bioRxiv preprint doi: https://doi.org/10.1101/2020.05.15.098863; this version posted May 16, 2020. The copyright holder for this preprint (which was not certified by peer review) is the author/funder. All rights reserved. No reuse allowed without permission.

\title{
Habitat loss causes long transients in small trophic chains
}

\author{
Blai Vidiella, ${ }^{1,2, *}$ Ernest Fontich, ${ }^{3,4}$ Sergi Valverde, ${ }^{5}$ and Josep Sardanyés ${ }^{6,4, \dagger}$ \\ ${ }^{1}$ ICREA-Complex Systems Lab, Universitat Pompeu Fabra, Dr Aiguader 88, 08003 Barcelona \\ ${ }^{2}$ Institut de Biologia Evolutiva, CSIC-UPF, Pg Maritim de la Barceloneta 37, 08003 Barcelona \\ ${ }^{3}$ Departament de Matemàtiques i Informàtica (Universitat de Barcelona). Gran Via 585, 08007 Barcelona, and Barcelona \\ ${ }^{4}$ Graduate School of Mathematics (BGSMath), \\ Campus de Bellaterra. Edifici C, 08193 Cerdanyola del Vallès, Barcelona. \\ ${ }^{5}$ Evolution of Technology Lab, Institute of Evolutionary Biology (CSIC-UPF). Pg. Barceloneta 37, 08003 Barcelona. \\ ${ }^{6}$ Centre de Recerca Matemàtica (CRM), Campus de Bellaterra. Edifici C, 08193 Cerdanyola del Vallès, Barcelona.
}

\begin{abstract}
Transients in ecology are extremely important since they determine how equilibria are approached. The debate on the dynamic stability of ecosystems has been largely focused on equilibrium states. However, since ecosystems are constantly changing due to climate conditions or to perturbations such as the climate crisis or anthropogenic actions (habitat destruction, deforestation, or defaunation), it is important to study how dynamics can proceed till equilibria. In this contribution we investigate dynamics and transient phenomena in small food chains using mathematical models. We are interested in the impact of habitat loss in ecosystems with vegetation undergoing facilitation. We provide a thorough dynamical study of a small food chain system given by three trophic levels: vegetation, herbivores, and predators. The dynamics of the vegetation alone suffers a saddle-node bifurcation, causing extremely long transients. The addition of a herbivore introduces a remarkable number of new phenomena. Specifically, we show that, apart from the saddle node involving the extinction of the full system, a transcritical and a supercritical Hopf-Andronov bifurcation allow for the coexistence of vegetation and herbivores via non-oscillatory and oscillatory dynamics, respectively. Furthermore, a global transition given by a heteroclinic bifurcation is also shown to cause a full extinction. The addition of a predator species to the previous systems introduces further complexity and dynamics, also allowing for the coupling of different transient phenomena such as ghost transients and transient oscillations after the heteroclinic bifurcation. Our study shows how the increase of ecological complexity via addition of new trophic levels and their associated nonlinear interactions may modify dynamics, bifurcations, and transient phenomena.
\end{abstract}

Keywords: Bifurcations - Facilitation - Habitat destruction - Nonlinear dynamics - Theoretical Ecology Long transients - Trophic chains - Scaling laws

\section{INTRODUCTION}

Ecosystems are highly nonlinear, complex dynamical systems [1-5]. Nonlinearities in ecology, driven by density-dependent interactions such as competition, cooperation, or victim-exploiter dynamics, introduce far from trivial cause-effects in the population dynamics. This is of special importance when dealing with ecosystems' responses to perturbations, both natural or of anthropogenic origin i.e. deforestation, habitat destruction, animal hunting. Nonlinear dynamics in ecosystems include intrinsic oscillations $[6,7]$ or deterministic chaos. For instance, chaos has been suggested to be found in vertebrate populations [8-12], plankton dynamics [13, 14], and insect species [15-18].

Nonlinearities also give place to TIPPING POINTS, which have important consequences in species persistence and extinctions. In the last years, tipping points are becoming extremely relevant in ecology, due to the anthropogenic effects on the ecosystem (i.e. climate change). Not only individual ecosystems can develop an abrupt shift (from glass melting to cyanoblooms) [19, 20], the whole planetary biome may

*Corresponding author: B. Vidiella (blai.vidiella@upf.edu). ORCID: 0000-0002-4819-7047

†Corresponding author: J. Sardanyés (jsardanyes@crm.cat). ORCID: 0000-0001-7225-5158 change [21-25]. One example of this abrupt change is the transition between the past vegetated Sahara to the current desert [26, 27]. In these ecosystems, the aridity restricts the availability to water, narrowing the vegetation cover. Moreover, external pressures like grazing or deforestation have shown that for the same aridity levels two possibilities are possible, vegetated or desert [28, 29]. In lower levels of aridity multiple ecosystems can be observed (savanna, forest, grasslands, and shrublands) but for some levels of aridity abrupt changes occur [30]. Furthermore, in the framework of systems biology, a tipping point driving populations to extinction has been recently described experimentally in yeast [31].

Tipping points can be studied by means of bifurcation theory (Box 1). It is important to note that long transients typically arise near bifurcations, where slowing down phenomena take place. Of particular interest are the so-called SADDLE REMNANT or GHOSTS, suggested as transient-generator mechanisms in ecological systems in Ref. [32]. Ghosts arise in biological dynamical systems with strong feedbacks such as cooperation [33-35] and facilitation processes [36, 37] (see below). Other suggested mechanisms responsible for transients are chaotic saddles responsible for transient chaos [38, 39], spatial systems [40], linear systems with varying time scales, coupled oscillators, and stochasticity [41] (see also Refs. [42-44]). 

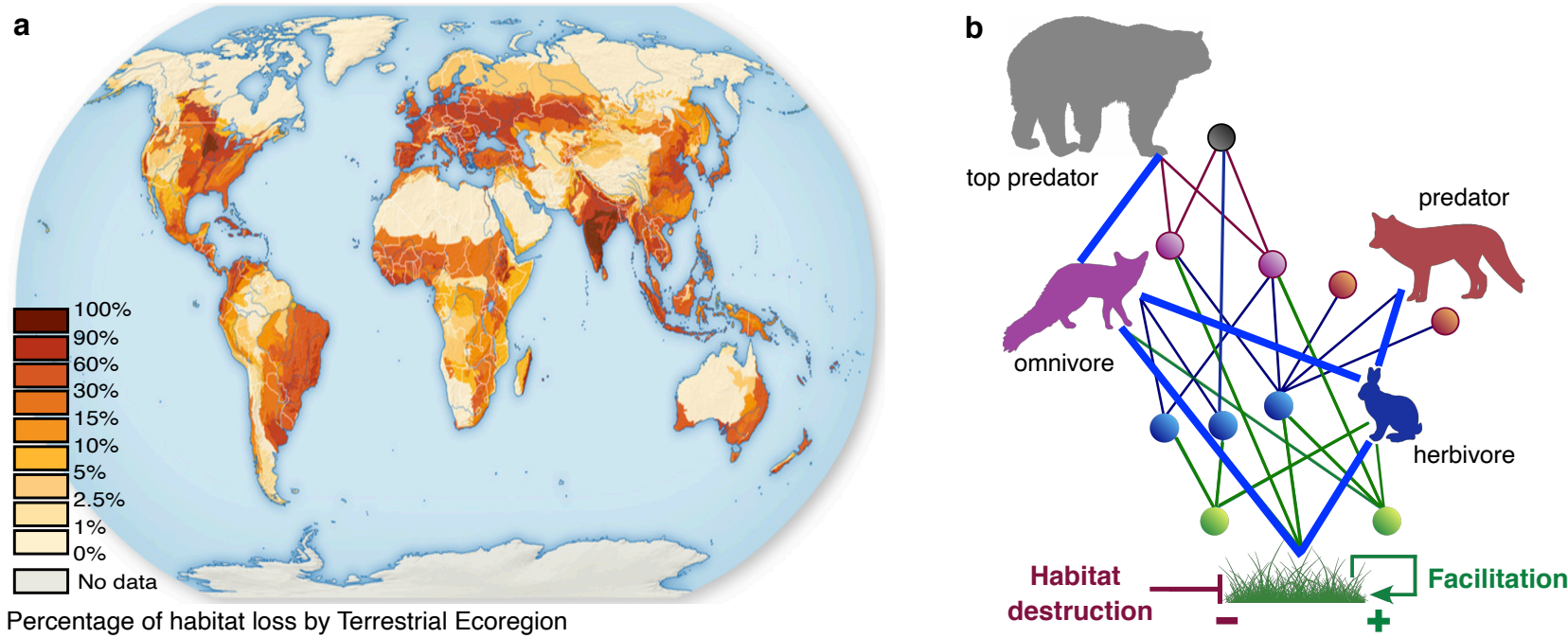

FIG. 1: (a) Habitat loss is an important ecological handicap for species persistence. Here we display the percentages of habitat loss divided in terrestrial eco-regions (source: http://habitatlossfragmentation.weebly.com/habitat-loss.html). (b) Trophic networks underly ecosystem dynamics. Here we display a large trophic network with different trophic levels: primary producers (green); herbivores (blue); omnivores (violet); predators (brown and grey). In this manuscript we will address the subnetwork highlighted by blue thick links by means of a simple dynamical model, focusing on the interactions vegetation-herbivore-predator and considering facilitation processes among primary produces and habitat destruction. Finally, the model model adding an omnivore and a top predator will be introduced. Our main goal is to characterise how an increase in ecological complexity (addition of different trophic levels) impacts on the bifurcations and the transients.

Recent research shows how human activity could be pushing ecosystems towards tipping points [45, 46]. Habitat fragmentation is one of the major causes of species extinctions [47-50]. The loss of wild areas [51] is increasing in the last years, also making species extinctions more and more frequent, leading to the so-called sixth mass extinction [52-56]. This, among other anthropogenic alterations, can lead to run-away effects from the bio-geo-physical planetary processes $[19,20,25]$ e.g. when grass disappears the soil is exposed to wind erosion, impairing the establishment of grass again [30]. In this sense, habitat destruction plays a major role in determining the fate of ecosystems. Figure 1(a) shows the planetary distribution of fragmented habitats, which comprise the most developed countries as well as the currently developing ones. Several works have studied the impact of habitat destruction with simple models [37, 50, 57], giving clues on the expected dynamics and bifurcations.

\section{Glossary}

Tipping point: qualitative change in dynamics produced by changes in parameter/s (equivalent to bifurcation).

Equilibrium or fixed point: state of the dynamical system that does not move as time changes. It satisfies $d x / d t=0$ for a single variable system. It can be either stable or unstable.

Phase space: set of all possible states of the dynamical system. This space is built using the dynamical variables as axes.

Eigenvalues of a fixed point: factors of contraction or expansion along their associated invariant directions (generated by the corresponding eigenvectors) of the lin- earized system at the fixed point. They are used to determine the stability of equilibrium points.

Orbit: path described by the dynamical variables in the phase space as time changes.

Saddle-node remnant (ghost): effect found close to a saddle-node bifurcation after the equilibrium points have disapeared which causes extremely long transients [32, 33, 35, 60]. Identified in electronic circuits [61].

Delayed transition: slowing down for saddle-node bifurcations casued by the ghost.

Monostability: dynamical system with a single stable equilibrium.

Bistability: dynamical system with two stable equilibria. Classic ecological examples are shallow and eutrophic lakes $[62,63]$, vegetated or desert states in semiarid ecosystems[28, 30] (see also [64]).

Globally asymptotically stable equilibrium point: equilibrium point that is stable and such that all orbits of the system converge to it.

Focus: equilibirum point for which all nearby orbits describe a spiral behaviour, either stable or unstable.

Stable limit cycle:periodic oscillation which attracts all nearby orbits.

Strange attractors: attracting objects in phase space with complicated geometric structure (fractal). Usually the dynamics on them is chaotic.

Stable manifold of an equilibrium: set of points that converge to the equilibrium, usually having less dimension than the one of the system. In the plane they are curves that separate regions with different dynamical behavior.

Unstable manifold: same as the stable manifold but with backward time.

Heteroclinic connection: set of orbits connecting two 
different equilibrium points of saddle type.

In this article we investigate a simple mathematical model describing the dynamics of a small trophic chain including facilitation at the level of primary producers and habitat destruction/loss. Our interest is to see how increasing ecological complexity i.e., the addition of trophic levels and interactions, affects dynamics, focusing on bifurcations and transients. The interplay between the triad habitat loss-facilitation-trophic relations has not been investigated in detail, especially at the level of transients. Facilitation (positive pairwise interactions between individuals leading to the benefit of at least one of the interacting partners[58]) is a key ecological process in many ecosystems e.g. drylands. This positive effect can occur either directly or indirectly. An example of the former includes shading mechanisms that reduce water or nutrient stress. The later would include removing competitors or deterring predators [59].

To ease the reading to an audience non familiarised with technical mathematical details, we provide a glossary of words and a box gathering the main characteristics of the bifurcations identified in our work.

\section{MATHEMATICAL MODEL AND RESULTS}

\section{Model 1. Vegetation with facilitation}

Starting with the simplest system we aim at characterising how the increase in ecological interactions and the addition of species impact dynamics, bifurcations, and, especially, transients. The first model describes a population of primary producers (e.g., plants, cyanobacteria, algae) with facilitated reproduction and intra-specific competition, together with habitat destruction and density-independent death rate. Facilitation is introduced with the nonlinear term $\alpha V^{2}$, $\alpha$ being the intrinsic growth rate of plants. This growth kinetics has been used to model autocatalysis [32] and ecological facilitation [37], in which a replicator enhances its own growth. As a difference from exponential growth, $\alpha V$, the autocatalytic one is known to produce hyperbolic dynamics, in which an infinite population can be achieved in finite time. This can be seen from the time solution for $\dot{V}=\alpha V^{2}$, given by $V(t)=V(0) /(1-V(0) \alpha t)$.

In our approach, the reproduction of the vegetation is constrained by a logistic-like function $1-(D+$ $V) / C_{0}$, where constant $D$ is the fraction of habitat destroyed (see Refs. [37, 50, 57, 65, 66]) and $C_{0}$ is the carrying capacity (set to 1 for simplicity). Hence, this first model considers four main ecological processes: (i) reproduction of plants with facilitation proportional to parameter $\alpha>0$, (ii) habitat destruction modelled with parameter $D \in[0,1]$, (iii) intraspecific competition, and (iv) density-independent death rate $(\epsilon)$ of plants. Parameter $D$ takes values between 0 and 1 since it represents the (normalised) fraction of

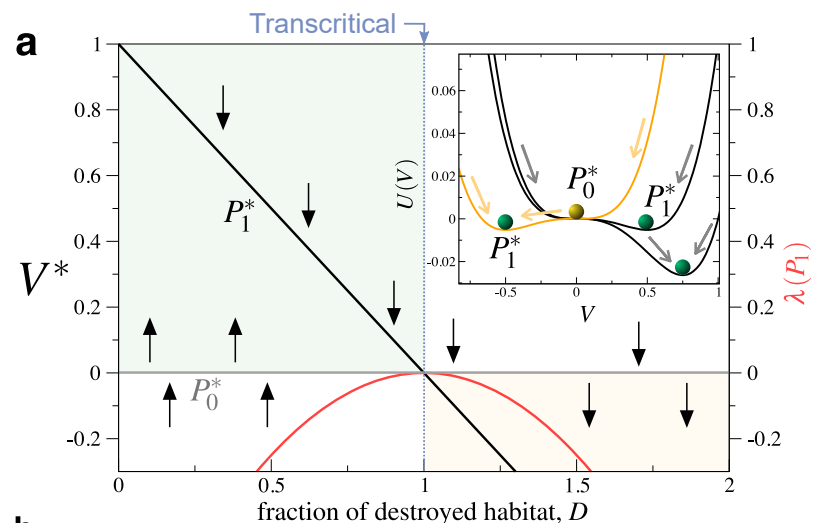

b

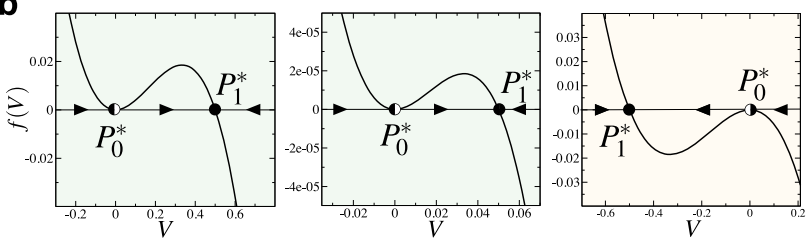

FIG. 2: Dynamics of Eq.(1) with $\epsilon=0$. (a) Equilibrium values of the vegetation at increasing the fraction of habitat destroyed, $D$, given by $P_{0}^{*}=0$ and $P_{1}^{*}=1-D$. The green rectangle displays the biologically-meaningful values of $D$, with $0 \leq D \leq 1$. Notice that within this range the fixed point $P_{0}^{*}$ is unstable (small vertical arrows denote the stability). Also, $P_{1}^{*}$ is always stable except at the bifurcation value $D=1$, where $\lambda\left(P_{0}^{*}\right)=\lambda\left(P_{1}^{*}\right)=0$ (the red curve shows the value of $\left.\lambda\left(P_{1}^{*}\right)\right)$. This means that transients towards e.g. $P_{1}^{*}$, become extremely long as $D \rightarrow 1$. The inset in (a) displays the potential function (2) computed with $D=0.25$ and $D=0.5$ (black lines), and $D=1.5$ (orange line). Notice that $P_{0}^{*}$ is half-stable: repeller for $V(0)>0$ and $0 \leq D<1$. At $D=1$ this stability for $P_{0}^{*}$ is changed. (b) Flows on the line displayed in the space $(f(V), V)$ : (left) $D=0.25$; (middle) $D=0.95$; (right) $D=1.5$ (here black dots indicate stable equilibria while black-white dots denote half-stable points). In all panels we have set $\alpha=1$.

habitat destroyed. The model is given by:

$$
f(V)=\frac{d V}{d t}=\alpha V^{2}(1-D-V)-\epsilon V
$$

The previous model has been recently studied in Ref. [37], and will be briefly discussed below. Let us first study the simplest model for vegetation setting $\epsilon=0$. This system has two EQUILIBRIUM or FIXED POINTS, obtained from $\dot{x}=0$, with $P_{0}^{*}=0$ and $P_{1}^{*}=$ $1-D$. The local stability of these equilibria can be computed from $\lambda=d f(V) / d V=2 \alpha V(1-D-3 / 2 V)$. It is easy to see that $\lambda\left(P_{0}^{*}\right)=0$ and that $\lambda\left(P_{1}^{*}\right)<0$ under the assumption that $0<D<1$, thus $P_{1}^{*}$ being stable within this range of $D$. This can be seen in Fig. 2(a), where the dynamics of Eq.(1) have been computed numerically[114] setting $\epsilon=0$. The population declines linearly as $D$ increases. The stability of this equilibrium, computed from $\lambda\left(P_{1}^{*}\right)$ is indicated with a red line, which is equal to zero at $D=1$.

The case $\lambda\left(P_{0}^{*}\right)=0$ does not provide any information on the stability of the origin. To determine the stability of this equilibrium we will compute a potential function and use graphical methods. A useful way to study and visualise the stability of the fixed points 
in a one-variable system is by means of the potential function, $U(V)$, given by:

$$
U(V)=-\int f(V) d V=\alpha V^{3}\left(\frac{V}{4}-\frac{1}{3}(1-D)\right) .
$$

The inset in Fig. 2(a) displays the potential, which has a minimum at $P_{1}^{*}$. Note that the origin is semistable. This can be clearly seen plotting $f(V)$ against $V$ (Fig. 2(b)). Due to the simplicity of this model one can compute the transient times to the fixed point $P_{1}^{*}$ analytically, from $d V / d t=f(V)$, using:

$$
d t=\frac{d V}{f(V)}, \quad \text { and } \quad \int_{t_{0}}^{t} d t=\int_{V(0)}^{V(t)} \frac{d V}{f(V)},
$$

obtaining, taking $t_{0}=0$ :

$$
t=\frac{1}{\alpha(D-1)^{2}}\left[\ln \frac{V}{1-D-V}+\frac{D-1}{V}\right]_{V(0)}^{V(t)} .
$$

From the previous expression it is easy to see that $t \rightarrow \infty$ as $D$ tends to the bifurcation value.

As we previously mentioned, the dynamics of Eq. (1) with $\epsilon>0$ has been recently investigated [37]. This system has two different dynamical regimes (MONOSTABILITY or BISTABILITY) as a function of the model parameters. This model has three equilibrium point, namely $P_{0}^{*}=0$ and the pair

$$
P_{ \pm}^{*}=\frac{1}{2}\left(1-D \pm \sqrt{(1-D)^{2}-4 \epsilon / \alpha}\right)
$$

Notice that the pair $P_{ \pm}^{*}$ will be biologically meaningful whenever the discriminant of the fixed points (4) is either positive or zero. At the bifurcation value the discriminant is zero and these two fixed points collide in a saddle-node bifurcation. This occurs at

$$
D_{c}=1-2 \sqrt{\epsilon / \alpha} \text {. }
$$

Linear stability analysis for this system indicates that when $D<D_{c}$ both $P_{0}^{*}$ and $P_{+}^{*}$ are locally asymptotically stable, while $P_{-}^{*}$ is a repeller. At $D=D_{c}, P_{+}^{*}$ and $P_{-}^{*}$ collide and are destroyed, and thus the only remaining equilibrium is $P_{0}^{*}$, which becomes GLOBALLY ASYMPTOTICALLY STABLE [37]. The bifurcation diagram using $D$ as control parameter is shown in Fig. 3(a). It is known that just after a saddlenode bifurcation, transients experience an extremely long delay, called a DELAYED TRANSITION [60, 67]. This is due to a SADDLE REMNANT or GHOST that attracts the orbits towards the region of the PHASE SPACE where the collision occurred, although no fixed points are present. This is why this is called a ghost, phenomenon that was claimed as a transient generator mechanism in ecological systems in Ref. [32]. It is known that the passage time, $t_{p}$, through the ghost follows a universal scaling law of the form $t_{p} \sim$ $\left(D-D_{c}\right)^{-1 / 2}$, as panel (b) in Fig. 3 shows. This ghost can be easily observed in time series, where trajectories settle onto a flat, extremely long bottleneck before a rapid collapse (Fig. $3(\mathrm{~d}))$.
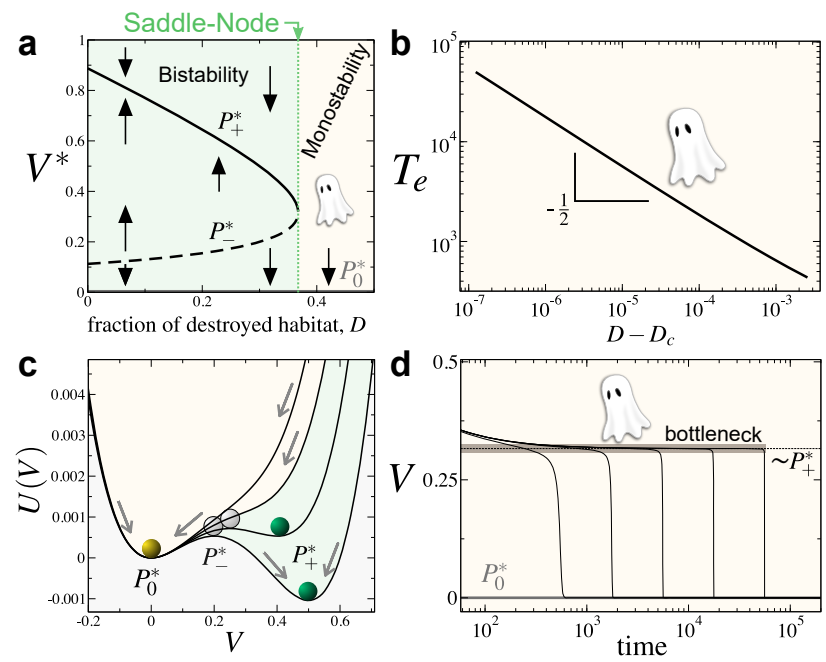

FIG. 3: Dynamics of Eq. (1) with $\epsilon>0$. (a) Saddlenode bifurcation, which leaves a ghost after bifurcation threshold and the system becomes monostable (yellow region). (b) Inverse square-root scaling law tied to the extinction transients trapped by the ghost. (c) Potentials for $D=0.3, D=0.35, D=0.4$, and $D=0.45$. In all panels we have used $\alpha=1$ and $\epsilon=0.1$. (d) Delayed transitions on the ghost bottleneck with $D=D_{c}+\omega$, with (from left to right): $\omega=10^{-3}, \omega=10^{-4}, \omega=10^{-5}, \omega=10^{-6}$, $\omega=10^{-7}$.

A potential function can be also computed for this system, now having:

$$
U(V)=-\alpha V^{2}\left(-\frac{\epsilon}{2 \alpha}+\frac{V(1-D)}{3}-\frac{V^{2}}{4}\right) .
$$

The potential is displayed in Fig. 3(c) for several values of $D$. For $D<D_{c}$ (solid lines) two wells are found, corresponding to the two stable states $\left(P 0^{*}\right.$ and $P_{+}^{*}$ ) resulting in bistability, that will be achieved depending on the initial conditions. Evidences of multiple states have been recently identified in semi-arid ecosystems $[28,30]$. Once the fraction of habitat destroyed surpasses its critical value $D_{c}$, a single well is found. This single stable state is given by the equilibrium point $P_{0}^{*}=0$, which involves extinction.

\section{Box 1: Bifurcations and their transients}

Bifurcations involve qualitative changes in dynamics when one or more parameters are changed [60, 112]. They can be generically classified as local or global. Local bifurcations involve fixed points (stability changes, collisions). Global bifurcations involve transitions associated to bigger objects such as global manifolds, limit cycles or STRANGE ATTRACTORS. Bifurcations are key phenomena in transients since they typically involve long delays of the orbits when parameters are close to bifurcation values. The bifurcations identified in this article are explained as follows. 


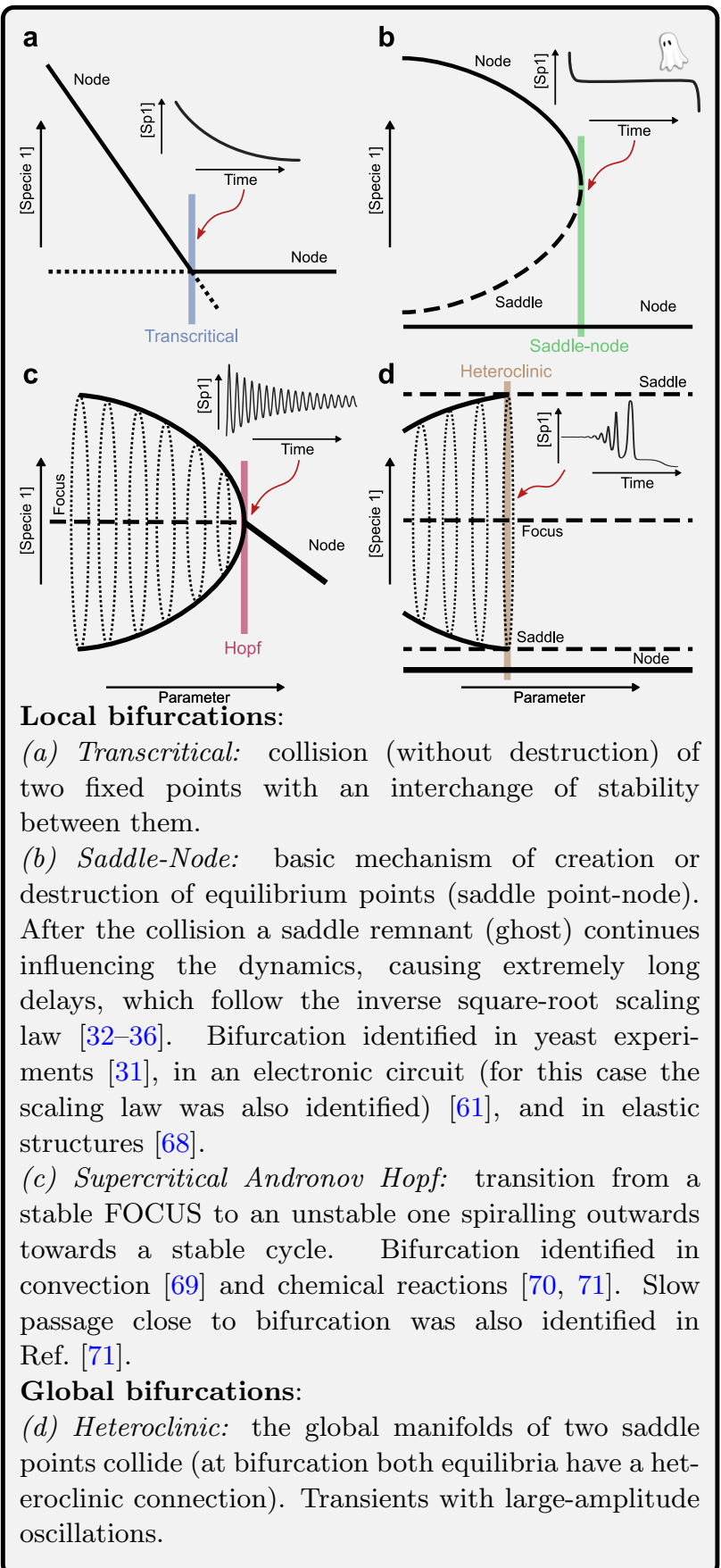

\section{Model 2. Vegetation-herbivore system}

In this section we consider another trophic level by adding a herbivore species to the dynamics of vegetation. That is, considering model (1) plus the new species, $S_{1}$. The model now reads:

$$
\begin{aligned}
\frac{d V}{d t} & =\alpha V^{2}(1-D-V)-\epsilon V-\epsilon_{1} V S_{1}, \\
\frac{d S_{1}}{d t} & =\mu_{1} \epsilon_{1} V S_{1}-\delta_{1} S_{1},
\end{aligned}
$$

Here we consider that vegetation is consumed by the herbivore at a rate $\epsilon_{1}>0$. Also, we consider that $S_{1}$ reproduces proportionally to $\mu_{1} \epsilon_{1}$, where $\mu_{1}<1$ is the effective reproduction rate. For simplicity $\mu_{1}$ and $\delta_{1}$ will be labeled as $\mu$ and $\delta$. The equilibrium points of Eqs. (6)-(7) are:

$$
\begin{aligned}
P_{0}^{*} & =(0,0) \\
P_{1 \pm}^{*} & =\left(P_{ \pm}^{*}, 0\right) \\
P_{2}^{*} & =\left(\frac{\delta}{\mu \epsilon_{1}}, \frac{\alpha \delta}{\mu \epsilon_{1}^{2}}\left(1-D-\frac{\delta}{\mu \epsilon_{1}}\right)-\frac{\epsilon}{\epsilon_{1}}\right)
\end{aligned}
$$

Note that equilibrium $P_{1 \pm}^{*}$ involves the extinction of the herbivore species and the persistence of vegetation, with same equilibria as the ones given in Eq. (4). Equilibrium $P_{2}^{*}$ involves coexistence. This equilibrium will be biologically-meaningful i.e. positive, when the fraction of habitat destruction is below the critical value:

$$
D_{c}=1-\left(\frac{\delta}{\mu \epsilon_{1}}+\frac{\mu \epsilon_{1} \epsilon}{\alpha \delta}\right) .
$$

The stability of the equilibrum points is computed from the linearised system given by the Jacobian matrix:

$$
\begin{gathered}
J\left(P^{*}\right)= \\
\left(\begin{array}{cc}
2 \alpha V\left(1-D-\frac{3}{2} V\right)-\epsilon_{1} S_{1}-\epsilon & -\epsilon_{1} V \\
\mu \epsilon_{1} S_{1} & \mu \epsilon_{1} V-\delta
\end{array}\right) .
\end{gathered}
$$

The stability of an equilibrium $\left(V^{*}, S_{1}^{*}\right)$ is computed from the sign of the EIGENVALUES obtained from the characteristic equation $\lambda\left(V^{*}, S_{1}^{*}\right)=\operatorname{det} \mid J\left(V^{*}, S_{1}^{*}\right)-$ $\lambda I \mid=0, I$ being the identity matrix. The eigenvalues of $J\left(P_{0}^{*}\right)$ are $\lambda_{1}=-\epsilon, \lambda_{2}=-\delta$, meaning that the origin is always a local attractor. The Jacobian matrix evaluated at equilibrium $P_{2}^{*}$ is:

$$
J\left(P_{2}^{*}\right)=\left(\begin{array}{cc}
\frac{\alpha \kappa}{\epsilon_{1}}\left(1-D-2 \frac{\kappa}{\epsilon_{1}}\right) & -\kappa \\
\frac{\alpha \delta}{\epsilon_{1}}\left(1-D-\frac{\kappa}{\epsilon_{1}}\right)-\mu \epsilon & 0
\end{array}\right),
$$

with trace

$$
\tau=\frac{\alpha \kappa}{\epsilon_{1}}\left(1-D-2 \frac{\kappa}{\epsilon_{1}}\right),
$$

and determinant

$$
\Delta=\frac{\alpha \delta \kappa}{\epsilon_{1}}\left(1-D-\frac{\kappa}{\epsilon_{1}}\right)-\delta \epsilon .
$$

We note that $\Delta>0$ in the range of parameters where $P_{2}^{*}$ exists . The stability of this fixed point can be determined with the sign of the trace, being an attractor when $\tau<0$, and a repeller when $\tau>0$. The bifurcation value $D_{c}^{(h)}$, involving the SUPERCRTICAL Andronov Hopf bifurcation, is here given by:

$$
D_{c}^{(h)}=1-2 \frac{\kappa}{\epsilon_{1}} .
$$

$P_{2}^{*}$ is stable when $D>D_{c}^{(h)}$. Since $\Delta>0 P_{2}^{*}$, is a focus. According to the previous calculations, the following local bifurcations are found in Eqs. (6)-(7) (see Fig. 4 and Box 1):

1. Saddle-node bifurcation identified in Section 2.1., causing delayed transitions (ghost transients). 
bioRxiv preprint doi: https://doi.org/10.1101/2020.05.15.098863; this version posted May 16, 2020. The copyright holder for this preprint (which was not certified by peer review) is the author/funder. All rights reserved. No reuse allowed without permission.
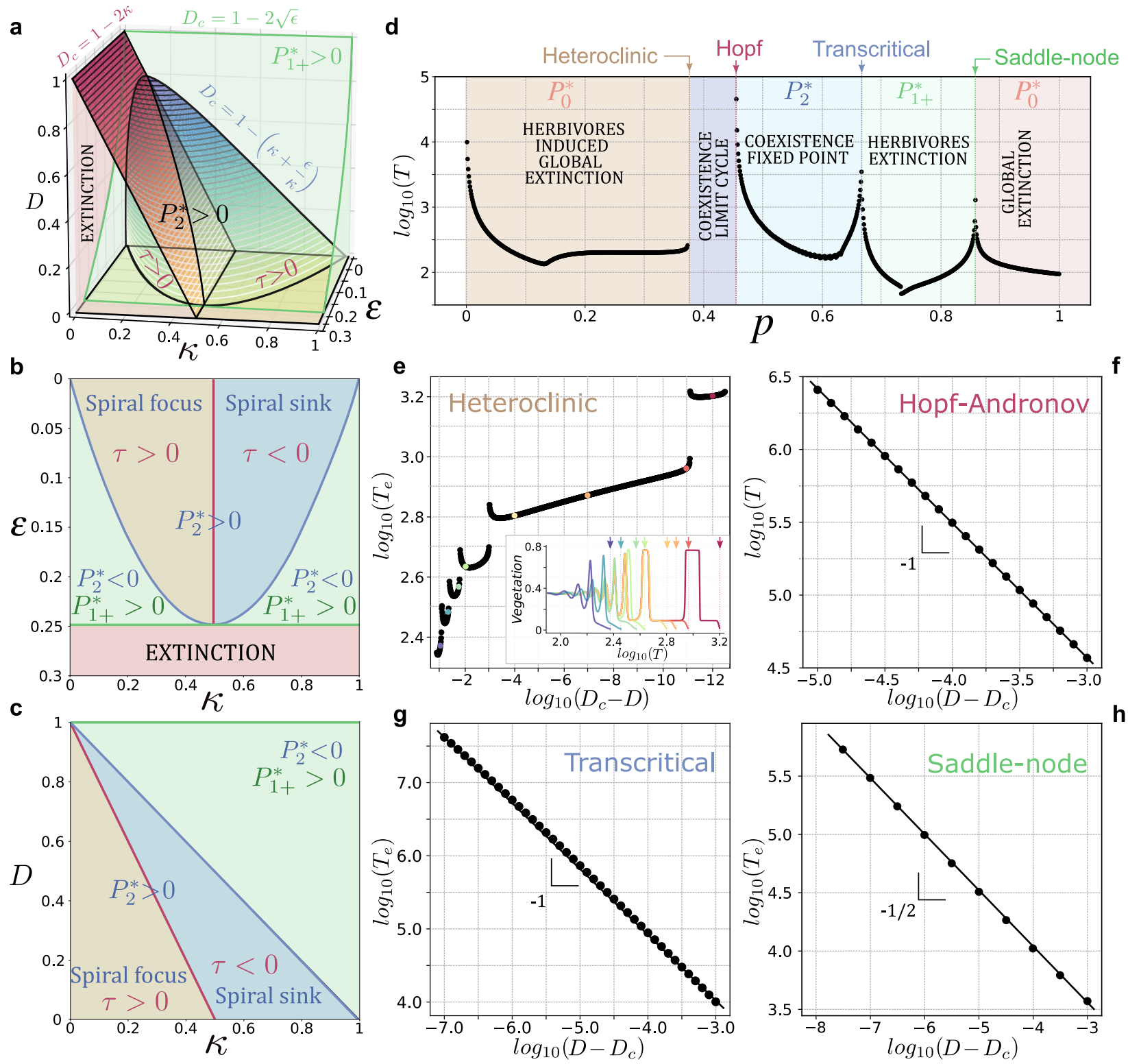

FIG. 4: Stability conditions, bifurcations,and transients of Eqs. (6)-(7). (a) Parameter space built with $(\epsilon, \kappa, D)$ showing the existence regions of equilibria and the boundaries involving bifurcations: under the green surface $P_{1 \pm}$ exist, under the blue one $P_{2}^{*}$ exists and their eigenvalues have imaginary part $(\Delta>0)$. The red surface indicates the change in $P_{2}^{*}$ stability (being a repeller below the surface). (b) Stability regions found in the plane $D=0$ (without habitat destructed). (c) Transitions occurring in the plane $\epsilon=0$ (without density-independent death of vegetation). (d) Times needed to achieve an attractor (given by equilibria within each coloured region) tuning simultaneously parameters $(\epsilon, \kappa, D)$ by using $p \times(0.2 \epsilon, \kappa, 0.2 D)$, with $p \in[0,1]$. Panels (e) to (h) display how transients change as the bifurcation parameter $D$ approaches to the bifurcation value $D_{c}$. (e) Transients close to the heteroclinic bifurcation. The inset displays several time series for different values of $D$ (indicated with the coloured dots).(f) Transient times before the SUPERCRITICAL HOF-ANDRONOV bifurcation of $P_{2}^{*}$ (red surface in the panel (a) and red lines in panels (b)-(c). (g) Transients arising after the the transcritical bifurcation of $P_{2}^{*}$ and $P_{1+}^{*}$ (blue surface in the panels (a)-(c). (h) Transients just after the saddle-node bifurcation giving place to the inverse square-root scaling law (green surface in panel (a)). In all the analyses $\alpha=\epsilon_{1}=1$.

2. Supercritical Andronov Hopf bifurcation when $P_{2}^{*}$ changes its stability from a stable focus to an unstable one (condition given by Eq. (14)). This will also induce long transients towards the oscillating coexistence.

3. Transcritical bifurcation when $P_{2}^{*}$ and $P_{1+}^{*}$ collide, occurring when $D=D_{c}$ (see Eq. (11)). This will cause a critical slowing down.

Equations (6)-(7) also suffer a global bifurcation given by a heteroclinic bifurcation originated from a heteroclinic connection between equilibria $P_{1+}^{*}$ and $P_{1-}^{*}$. This bifurcation occurs via the collision of the global manifolds of both fixed points (see Section IV A in the Appendix for detailed information). Close to the bifurcation, the geometric structure of the manifold forces all the trajectories to pass very close to the fixed points $P_{1 \pm}^{*}$ before achieving the origin, approaching the ORBITS to regions where the vector field is ex- 
bioRxiv preprint doi: https://doi.org/10.1101/2020.05.15.098863; this version posted May 16, 2020. The copyright holder for this preprint (which was not certified by peer review) is the author/funder. All rights reserved. No reuse allowed without permission.

tremely small thus causing delays and long transients. This actually causes a kind of slow-fast transient dynamics.

The dependence of dynamics on parameters and the bifurcations found in Eqs. (6)-(7) are summarised in Fig. 4: panels (a) shows the boundaries between the different dynamical regimes in the parameter space $(\epsilon, \kappa, D)$. Panels (b) and (c) display cuts in the threedimensional parameter space showing the stability of the equilibrium points and their existence. All of the bifurcations identified in the model are displayed in Fig. 4(d) tuning an auxiliary parameter $p \in[0,1]$, allowing to cross the parameter space in (a) with a straight line by means of $p \cdot(0.2 \epsilon, \kappa, 0.2 \delta)$, which is plotted against the times needed to achieve an attractor. Notice that transients increase near bifurcation values since the vector field tends to zero. It is known that the length of transients close to local bifurcations typically follow scaling relations between the parametric distance to the bifurcation value [60]. As we previously discussed, this scaling for saddlenode bifurcations is given by the inverse square-root scaling law [60].

These delays, known as delayed transitions for saddle-node bifurcations, are generically named as critical slowing down. Different exponents for these scaling laws are known to exist, being $-1 / 2$ for the saddle-node bifurcation and -1 for the transcritical bifurcation (see Fig. 4(h,g)). The supercritical Andronov Hopf bifurcation also displays a scaling exponent of -1 before the emergence of the STABLE LIMIT CYCLE, as shown in Fig. 4(f). As mentioned, the heteroclinic bifurcation is a global bifurcation, and involves changes in the global invariant manifolds, while fixed points neither change (remarkably) their stability nor collide. Figure 4(e) shows that, as the critical value of the heteroclinic bifurcation $D_{c}^{(H)}$ is approached, the time to extinction $T_{e}$ increases in a discontinuous way (the computation of $D_{c}^{(H)}$ can be found in Section IV B in the Appendix). These jumps are tied to the emergence of new cycles as $D^{(H)} \rightarrow D_{c}$. This can be clearly seen in Fig. 5 . Specifically, Fig. 5(a) shows a time series extremely close to the bifurcation value, where the dynamics undergoes 11 cycles[115] For some lower $D$ values the number of cycles diminishes, providing discontinuities in times as shown in Fig. 5(b) (see also Fig.4(e)). Figure 5(c) displays how the cycles appear and accumulate at increasing $D$.

We note that each new cycle forces the orbits to pass closer to the points $P_{1+}^{*}$ and $P_{1-}^{*}$, which are saddle points, and thus have stable and unstable local manifolds. This effect makes the cycles to lapse more time, giving to the time trajectories the typical square shape found in slow-fast systems (here in a transitory way, see also the inset time series in Fig.4(e)) as well as in asymptotic heteroclinic cycles dynamics (see e.g. [3, 72, 73]). Finally, we note that the exit time (the time of residence at the left side of $P_{1-}^{*}$ ) increases with the proximity to de critical parameter value. Figure 6 provides dynamics and bifurcations for the vegetation and herbivores, where each one of the local bifurcations as well as the global bifurcation
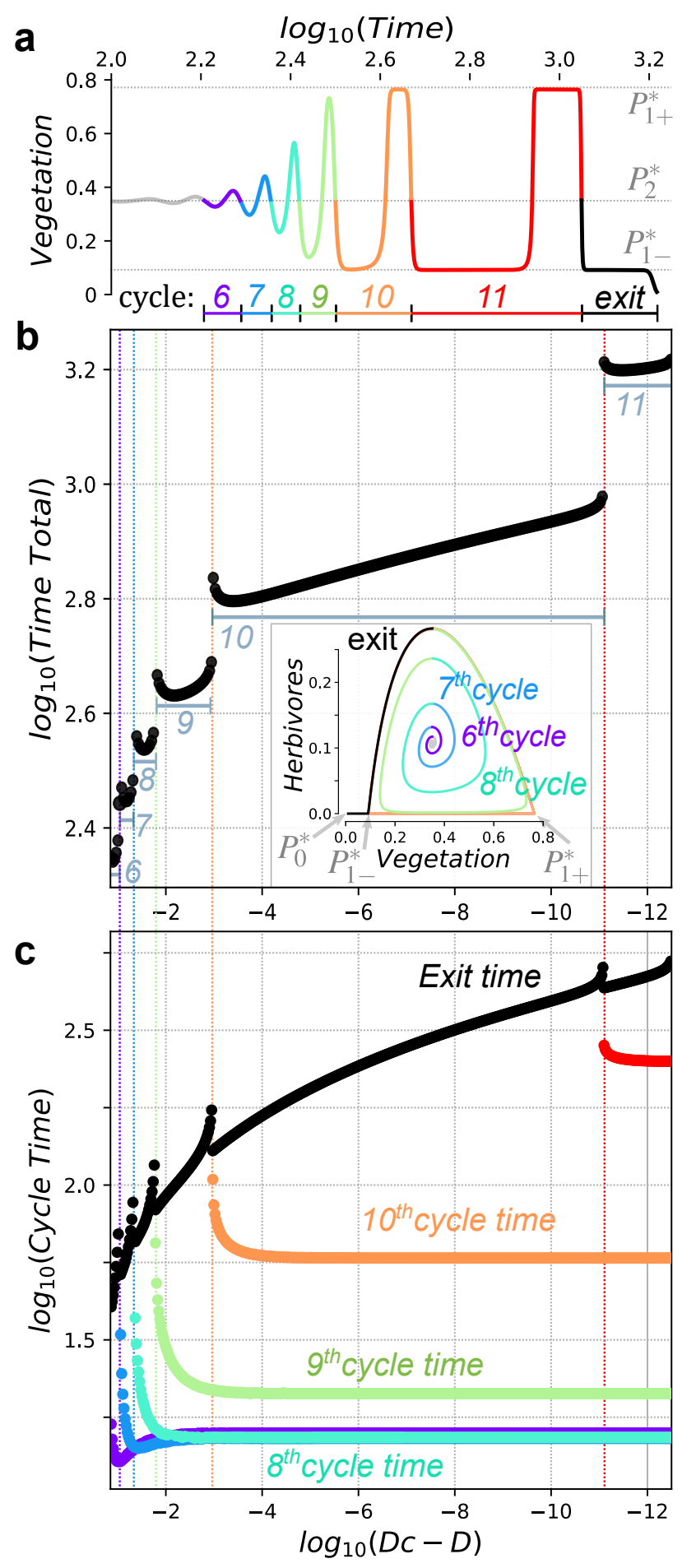

FIG. 5: Transients close to the heteroclinic bifurcation. (a) Extinction transients after the heteroclinic bifurcation with $D_{c}-D \sim 10^{-12}$. Each colour indicates a cycle around the repeller. (b) Same as in Fig. 4(e) now showing the trajectory represented in panel (a) in a phase portrait. Here the orbit gets very close to the saddle points $P_{1+}$ and $P_{1-}$. (c) Time per cycle depending on the distance to the bifurcation value. For these analysis we use: $\alpha=\mu=\epsilon_{1}=$ $1, \kappa=0.35, \epsilon=0.07$ and $D=0.1439373616781516 \lesssim D_{c}$. The initial conditions are taken near to $P_{2}^{*}$. 

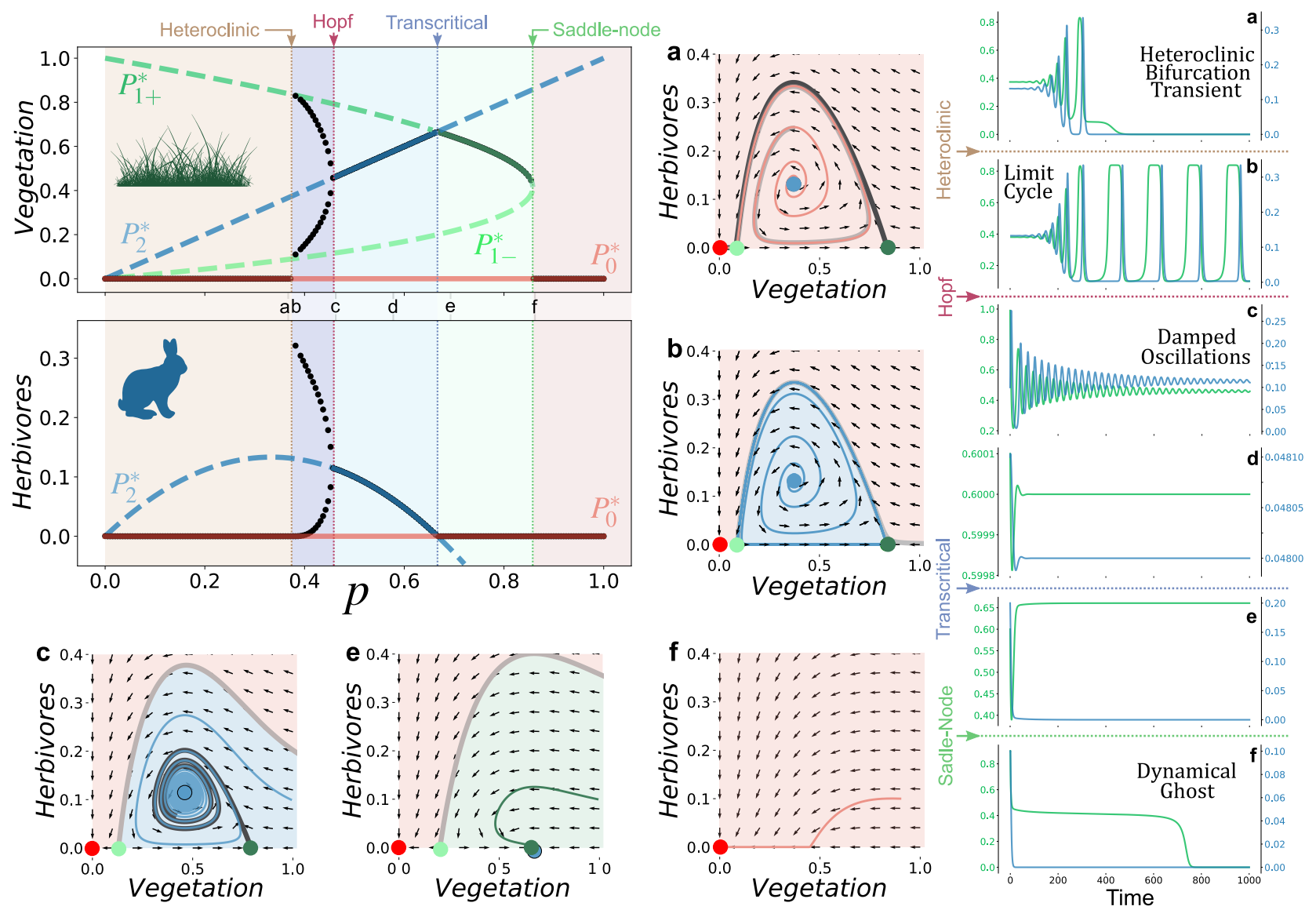

FIG. 6: Bifurcation diagrams tuning $p$ to sample the parameter space $(\epsilon, \kappa, D)$ with a straight line (more precisely, $D=\epsilon=0.2 p$ and $\kappa=p)$. The dynamics for different regions of the bifurcation diagrams are illustrated with the small letters showing phase portraits and time series. In the first region, $P_{0}^{*}$ is the only stable fixed point. Just after the heteroclinic bifurcation, a long transient with a slow-fast dynamics appear (see panels (a) with $p=0.3735$ ). Between the heteroclinic and the Hopf bifurcation, a limit cycle appears (panels (b. Right after the Hopf bifurcation, damped oscillations are obtained (see panels $\mathbf{c}$ with $p=0.46$ ), but they get largely tenuated when the parameter distance o the transition increases (panels $\mathbf{d}$ setting $p=0.6$ ). After the transcritical bifurcation the herbivores can not surviver, and vegetation remains in the ecosystem (panels $(\mathrm{e}), p=0.69$ ). Finally, when death rates are high enough, a saddle-node bifurcation occurs and all populations go to extinction (e.g. panels (f), $p=0.8582$ ). Equilibrium points in the phase portraits are shown with coloured dots: $P_{0}^{*}$ (red); $P_{1-}^{*}$ (light green); and $P_{1+}^{*}$ (dark green). Their basins of attraction are depicted by the coloured areas using same colours of equilibrium points. Global invariant manifolds for $P_{1-}^{*}$ and $P_{1+}^{*}$ are displayed with grey and black lines in the phase portraits. In all analyses we have used $\alpha=\mu=\epsilon_{1}=1$.

can be visualised. Each new dynamical regime upon bifurcation has an associated phase portrait and time series, represented in panels (a)-(f) in Fig. 6 .

Notice that all the described bifurcations, and their associated transients, can be achieved by increasing habitat destruction $(D)$, increasing the death rate of primary producers $(\varepsilon)$ i.e. deforestation/toxic compounds; increase of herbivores' hunting $(\delta)$ increasing $\kappa$; or raising their effective growth rate $(\mu)$ lowering $\kappa$. One example of this last possibility, may be the reduction in the abundance of higher trophic organisms (i.e. predators). The impacts of predators is studied in the following section.

\section{Model 3. Vegetation-herbivore-predator system}

In this section we investigate the impact of introducing a predator species, $S_{2}$, on the two-dimensional, vegetation-herbivore system previously studied. The model now reads:

$$
\begin{aligned}
\frac{d V}{d t} & =\alpha V^{2}(1-D-V)-\epsilon V-\epsilon_{1} V S_{1} \\
\frac{d S_{1}}{d t} & =\mu \epsilon_{1} V S_{1}-\epsilon_{2} S_{1} S_{2}-\delta S_{1} \\
\frac{d S_{2}}{d t} & =\rho \epsilon_{2} S_{1} S_{2}-\delta_{2} S_{2} .
\end{aligned}
$$

Here predators increase in numbers due to the consumption of the herbivores, $S_{1}$. The predation rate is $\epsilon_{2}>0$ and the effective reproduction of predators is given by the term $\rho \epsilon_{2}$, with $\rho<1$. Predators die at rate $\delta_{2}>0$. This dynamical system has seven equi- 



FIG. 7: Dynamics, bifurcations, and transients for Eqs. (15)-(17). (A) Dynamical and stability boundaries in the parameter space $(\kappa, \epsilon, D)$, fixing $\eta=0.01$. Relevant examples of dynamics are shown in the full phase space by means of phase portraits and time series for the regions indicated with small letters (a)-(f). (B) Minimal value of habitat destruction, $D$, needed to stabilise the dynamics at equilibrium $P_{2}^{*}$, allowing for the coexistence between the vegetation and the herbivore species (boundary given by Eq.(24) here with $\epsilon=0.1$ ). (C) Surface separating the full ecosystem coexistence governed by equilibrium $P_{3+}^{*}$, obtained from Eq. (23) (here setting $\kappa=0.35$ ). The colours of the orbits in panels (a)-(f) indicate the state achieved by the system: full extinction (red), vegetation-only persistence (green); vegetation-herbivores coexistence (blue); full species coexistence (black). Notice that the system can be monostable (panel (f)); bistable (panels (c), (e), and (d); and tristable (panels (a) and (b)). The values of the equilibrium points are shown with the dots in the phase space and the dashed lines in the time series. In all panels: $\alpha=\mu=\rho=\epsilon_{1}=\epsilon_{2}=1$.

librium points:

$$
\begin{aligned}
& P_{0}^{*}=(0,0,0), \\
& P_{1 \pm}^{*}=\left(P_{ \pm}^{*}, 0,0\right), \\
& P_{2}^{*}=\left(\frac{\delta}{\mu \epsilon_{1}}, \frac{\alpha \delta}{\mu \epsilon_{1}^{2}}\left(1-D-\frac{\delta}{\mu \epsilon_{1}}\right), 0\right), \\
& P_{3 \pm}^{*}=\left(\frac{(1-D)}{2} \pm \sqrt{\frac{(1-D)^{2}}{4}-\frac{\epsilon_{1} \delta_{2}}{\rho \epsilon_{2} \alpha}-\frac{\epsilon}{\alpha}}, \frac{\delta_{2}}{\rho \epsilon_{2}},\right. \\
& \left.\left.\frac{\mu \epsilon_{1}}{\epsilon_{2}}\left[\frac{(1-D)}{2} \pm \sqrt{\frac{(1-D)^{2}}{4}-\frac{\epsilon_{1} \delta_{2}}{\rho \epsilon_{2} \alpha}-\frac{\epsilon}{\alpha}}\right]-\frac{\delta}{\epsilon_{2}}\right) 2,1\right) \\
& P_{4}^{*}=\left(0, \frac{\delta_{2}}{\rho \epsilon_{2}},-\frac{\delta}{\epsilon_{2}}\right) .
\end{aligned}
$$

Note that, compared to the previous model, two new internal fixed points, $P_{3 \pm}^{*}$, appear $\left(P_{4}^{*}\right.$ is not biologically meaningful since it has a negative component).

Equilibrium $P_{3+}^{*}$ is now responsible for the coexistence of the full food chain. We note that fixed points $P_{3 \pm}^{*}$ will exist i.e., $P_{3 \pm}^{*} \in \mathbb{R}$, when $D<D_{c}$, with

$$
D_{c}=1-2 \sqrt{\frac{\epsilon}{\alpha}+\frac{\eta \epsilon_{1}}{\epsilon_{2} \alpha}}
$$

here with $\eta=\delta_{2} / \rho$. This means that equilibria $P_{3+}^{*}$ and $P_{3-}^{*}$ collide in a saddle-node bifurcation at $D=$ $D_{c}$, with $P_{3 \pm}^{*} \in \mathbb{C}$ for $D>D_{c}$.

The Jacobian matrix for this system is:

$$
\left(\begin{array}{ccc}
2 \alpha V\left(1-D-\frac{3}{2} V\right)-\epsilon_{1} S_{1}-\epsilon & -\epsilon_{1} V & 0 \\
\mu \epsilon_{1} S_{1} & \mu \epsilon_{1} V-\delta-\epsilon_{2} S_{3} & -\epsilon_{2} S_{1} \\
0 & \rho \epsilon_{2} S_{2} & \rho \epsilon_{2} S_{1}-\delta_{2}
\end{array}\right) .
$$

The equilibrium point placed at the origin $\left(P_{0}^{*}\right)$ still remains as a local attractor, with eigenvalues $-\epsilon,-\delta,-\delta_{2}$. Equilibria $P_{1 \pm}^{*}$, involving only the persistence of vegetation, gain a stable direction in the full phase space i.e., $\lambda_{3}=-\delta_{2}$. Finally, the vegetationherbivore species found in the phase plane $(V>$ $\left.0, S_{1}>0\right)$ gains an additional dimension, whose stability depends upon the critical value $D_{c}^{\prime}$,

$$
D_{c}^{\prime}=1-\left(\frac{\kappa}{\epsilon_{1}}+\frac{\epsilon \epsilon_{1}}{\alpha \kappa}+\frac{\eta}{\kappa} \frac{\epsilon_{1}^{2}}{\alpha \epsilon_{2}}\right)
$$

recall $\kappa=\delta / \mu$. For $D>D_{c}^{\prime}, P_{+}^{*}$ gains a stable direction in the full space. Comparing this to the stability condition (24) and the existence condition (11), it is easy to see that there is a parameter region where the fixed point is a repeller in the predator dimension and it turns to an attractor. This means that there is a 

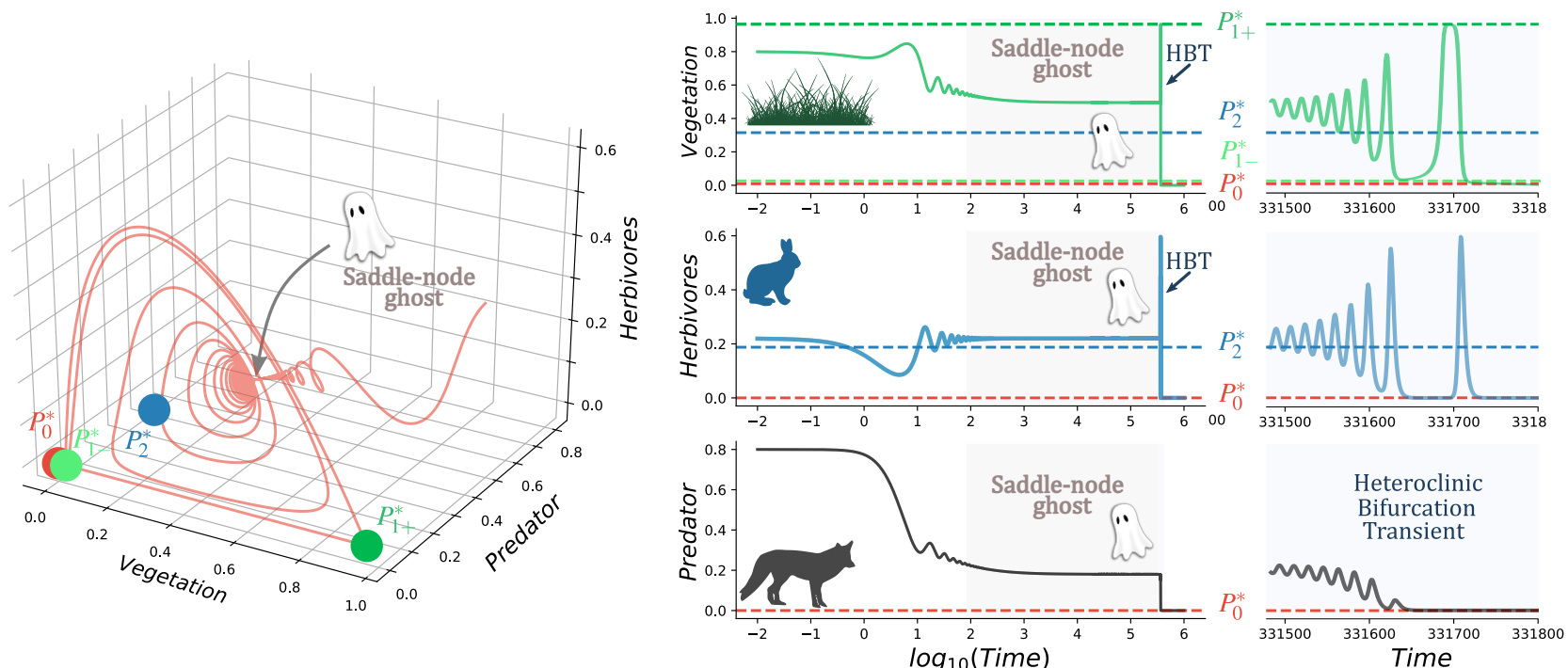

FIG. 8: Example of a chain of transients towards extinction visualised in the full phase space and with time series. (Left) Typical path followed by an orbit starting far away from the region of the phase space where equilibria $P_{3 \pm}^{*}$ collided, leaving a ghost. Such a region sucks the orbit, which remains trapped for an extremely long time, and then spirals outwards experiencing the delays when they pass close to the saddle points $P_{1 \pm}^{*}$ before going to the origin. (Right) Time series for vegetation, herbivores, and predators, with an initial oscillatory approach towards the ghost, a further delay settlet on the bottleneck region of the ghost (grey area), and the final large oscillations tied to the heteroclinic bifurcation transient (labelled as HBT). In all the analyses we use: $\epsilon=0.025, D=0.01, \alpha=\mu=\rho=1.0, \delta=0.315$, and $\delta_{2}=0.220025$.

region where tristability exists: the global extinction $\left(P_{0}^{*}\right)$, the global coexistence $\left(P_{4+}^{*}\right)$ and the coexistence of vegetation and herbivours. This combined with the results from the previous section (the dynamics when $S_{2}=0$ should be preserved from the $2 \mathrm{D}$ model) indicate the herbivores-vegetation attractor can be either the fixed point $P_{2}^{*}$ or the limit cycle (explained in the previous section) depending on the stability condition of $P_{2}^{*}$ (see Eq. (11)).

The possible dynamics and the bifurcation boundaries of this system are displayed in Fig. 7. Panel A shows these dynamical regimes, which include coexistence and extinctions, in the parameter space $(\kappa, \epsilon, D)$. To illustrate qualitatively different dynamics we have selected different regions of this parameter space (see Fig. 7(a-f)): (a) bistable system with coexistence of all the species $\left(P_{4+}^{*}\right.$ locally stable (LS)) and extinction $\left(P_{0}^{*} \mathrm{LS}\right)$; (b) tristability with coexistence of all the species $\left(P_{4+}^{*} \mathrm{LS}\right)$, herbivore-vegetation coexistence $\left(P_{2}^{*} \mathrm{LS}\right)$ and extinction $\left(P_{0}^{*} \mathrm{LS}\right) ;(\mathrm{c})$ bistable state, herbivore-vegetation coexistence $\left(P_{2}^{*} \mathrm{LS}\right)$ and extinction $\left(P_{0}^{*} \mathrm{LS}\right)$; (d) bistable state, herbivorevegetation coexistence governed by a limit cycle $\left(P_{2}^{*}\right.$ locally unstable) and extinction ( $\left.P_{0}^{*} \mathrm{LS}\right)$; (e) Bistable state $\left(P_{1+}^{*} \mathrm{LS}\right)$ and $\left(P_{0}^{*} \mathrm{LS}\right)$; and $(\mathrm{f})$ global extinction $\left(P_{0}^{*}\right.$ globally stable).Panels $\mathrm{B}$ and $\mathrm{C}$ show results for parameters $\kappa, \eta, \epsilon$, and $D$ concerning existence of fixed points. Figure 8 displays an example of how different transient phenomena can concatenate. Specifically, the orbit shown first approaches the saddlenode ghost via oscillations and, after the extinction of predators, follows the spiral dynamics towards the full extinction. Note that the main delay is here caused by the ghost. The time series in Fig. 8 illustrate how this transient causes the chain of extinctions (similar to the extinction debt of metapopulations $[50,74,75]$.

\section{Larger trophic chains: future research}

The models analysed in the previous sections can be extended to include further ecological complexity, as shown in Fig. 1(b). These simple models may also allow for a feasible investigation of the population dynamics on complex networks. Below, we propose two more models to be studied elsewhere. The first one adds an omnivore species that consumes the vegetation and predates on $S_{1}$. The later includes a top predator consuming the omnivore species

\section{Model 4. Vegetation, herbivores and omnivores}

This model considers the vegetation-herbivore species plus another species, $S_{3}$, consuming the vegetation and predating the omnivore species. The model reads:

$$
\begin{aligned}
\frac{d V}{d t} & =\alpha V^{2}(1-D-V)-\epsilon V\left(S_{1}+S_{3}\right), \\
\frac{d S_{1}}{d t} & =\mu \epsilon V S_{1}-\epsilon_{3} S_{1} S_{3}-\delta S_{1}, \\
\frac{d S_{3}}{d t} & =\mu_{3} \epsilon V S_{3}+\rho \epsilon_{3} S_{1} S_{3}-\delta_{3} S_{3} .
\end{aligned}
$$

Here vegetation is consumed by the herbivore $S_{1}$ and by $S_{3}$, which grows proportionally to the term $\mu_{3} \epsilon>$ 0 , here also with $\mu_{3}<1$. Omnivores are assumed to die proportionally to rate $\delta_{3}>0$. 
bioRxiv preprint doi: https://doi.org/10.1101/2020.05.15.098863; this version posted May 16, 2020. The copyright holder for this preprint (which was not certified by peer review) is the author/funder. All rights reserved. No reuse allowed without permission.

Model 5. Vegetation, herbivores, omnivores and a top predator

Here we consider the dynamics of the previous model adding a top predator, $S_{4}$, that consumes the omnivore species, with dynamical equations:

$$
\begin{aligned}
\frac{d V}{d t} & =\alpha V^{2}(1-D-V)-\epsilon V\left(S_{1}+S_{3}\right) \\
\frac{d S_{1}}{d t} & =\mu V S_{1}-\epsilon_{3} S_{1} S_{3}-\gamma S_{1} S_{3}-\delta S_{1} \\
\frac{d S_{3}}{d t} & =\mu_{3} V S_{3}+\rho \epsilon_{3} S_{1} S_{3}-\gamma S_{3} S_{4}-\delta_{3} S_{3}, \\
\frac{d S_{4}}{d t} & =\theta \gamma S_{4} S_{3}-\delta_{4} S_{4} .
\end{aligned}
$$

The reproduction rate of the top predator is proportional to $\theta \gamma>0$ and $\theta<1$, also assuming that they die proportionally to constant $\delta_{4}>0$.

\section{DISCUSSION}

We have studied simple dynamical models for small trophic chains. Our main goal was to characterise how the addition of new species and thus new ecological interactions affected the dynamics, focusing on bifurcation phenomena and transients. We considered processes of facilitation at the level of primary producers, focusing on the impact of habitat loss, suggested as a key factor jeopardising species survival [47-50]. First, we have introduced a simple model for vegetation with facilitation recently studied [37]. This system is known to suffer a saddle-node bifurcation giving place to an abrupt tipping point as the habitat destruction overcomes a critical threshold.

The addition of a species consuming the vegetation introduces important changes in the dynamics, now allowing for the coexistence of both species and extinction of the omnivores or of the entire system. Here we identify a transcritical bifurcation separating the coexistence of both species and the extinction of the herbivores. The coexistence regime can be achieved via stable fixed point or via limit cycle. Interestingly, a global bifurcation driven by a heteroclinic bifurcation can induce a global extinction, in which the two species become extinct (a scenario dynamically analogous to the one found after the saddle-node bifurcation). The transient times and their dependence on bifurcation threshold have been determined. The transients after the heteroclinic bifurcation change in a discontinuous fashion as the habitat destruction approaches the critical value.

The previous system has been extended by adding a predator species consuming the herbivores. The most remarkable result of this model is that different phenomena responsible for transients can get coupled, thus enlarging transients. For instance, for some parameter regions, a ghost caused by a saddle-node bifurcation can get coupled with the oscillatory transient of the vegetation-herbivores system once the heteroclinic bifurcation has occurred. The coupling of different transients arising from different mechanisms may be relevant in theoretical ecology and may deserve further attention in future research.

Our results reflect the importance of so-called trophic downgrading [64]. The increase of hunting pressures on top predators (larger $\delta_{2}$ and $\rho$ in our model), may cause a complete shift in dynamics, usually towards extinctions [76, 77]. During the transient, where all species remain, the reintroduction of predators could help in maintaining species and diversity, but effective changes may be introduced at the level of parameters (e.g. decreasing hunting of predators). The former strategy is the so-called rewilding [78-80]. Rewilding of wolfs in the Yellowstone National Park increased the complexity of the ecosystem, increasing the number of species living there and their abundances [81-83]. Once the wolfs became extinct, elks were out of control and they ate too much vegetation leading to lower $\mathrm{CO}_{2}$ capture and less food for other herbivores [83-85]. Moreover, the decrease in habitat space also led to simplifications of the trophic chain, even allowing only the persistence of producers. This is the so-called ecological meltdown, observed in some fenced areas in Africa [86-89]. For this case, what is being implemented are the formation of wildlife corridors [90-92], which increase the effective living area (meaning a decrease of habitat fragmentation parameter $D$ ). Notice that all the possible interventions should be done during the transients to ensure that the ecosystem is kept as similar as possible to the one found before multiple extinctions happened.

We note that the model explored here does not take into account complex functional responses [9397], which introduce more realism to predator-prey dynamics. Despite this issue, our model includes the minimal interactions between the different tropic levels, allowing a clear identification of how different threads can push the system towards different kinds of bifurcations which have different properties (i.e. delayed extinction time) and signatures (changes in the temporal dynamics, see Fig.6), similar to what is observed in real systems [31, 44, 98-100].

The presence of extremely long transients close to bifurcations (as shown in this article) opens the possibility that some ecosystems may be currently living in a ghost state, transitioning towards a less complex state $[76,77,101]$ due to impact of human activity including deforestation, environmental contamination or defaunation. Anthropogenic impacts are making ecosystems to experience defaunation [102, 103], suffering extinction cascades due to trophic downgrading or ecological meltdown caused by ecosystem domestication (i.e. hunting top predators or cultivation fields expansion) [104-107]. These transients, where some species may be in a slow declining regime, or even in a false apparent stationary state, should provide opportunities for restoring ecosystems. Restoration strategies may be implemented during the transients $[36,108]$ to recover the maximum number of species and the complete ecosystem function (i.e. carbon and nitrogen fixation $[109,110])$. More studies with real data should address this question. Also, future work may consider multiple species at each trophic layer [111], providing a closer approach to 
bioRxiv preprint doi: https://doi.org/10.1101/2020.05.15.098863; this version posted May 16, 2020. The copyright holder for this preprint (which was not certified by peer review) is the author/funder. All rights reserved. No reuse allowed without permission.

ecosystems as complex networks. Also, the impact of both intrinsic and extrinsic noise in the dynamics described in this contribution may be of interest.

\section{Acknowledgements}

BV wants to thank Alan Hastings and Kim Cuddington for the invitation to contribute to the Organized Oral Session on Transients in Ecology at the Ecological Society of America in 2019. The authors thank Ricard Solé and Tomás Lázaro for useful discussions.

Funding information $\mathrm{BV}$ has been funded by the PR01018-EC-H2020-FET-Open MADONNA project and by the Botin Foundation, by Banco Santander through its Santander Universities Global Division. SV was supported by the Spanish Ministry of Economy and Competitiveness, grant FIS2016-77447-R MINECO/AEI/FEDER and the European Union. JS has been partially funded by the CERCA Programme of the Generalitat de Catalunya, by the MINECO grant MTM-2015-71509-C2-1-R and the Spain's "Agencia Estatal de Investigación" RTI2018098322-B-I00, and by a "Ramón y Cajal" contract (RYC-2017-22243). EF has been partially supported by the Spanish Government grant MTM2016-80117-P (MINECO/FEDER, UE) and by the Catalan Government grant 2017-SGR-1374.

\section{Conflict of interest}

The authors declare that they have no conflict of interest.

\section{References}

[1] Volterra V (1926) Variazioni e fluttuazioni del numero da individui in specie animali convivienti. Memoroo Acadamei Linceii 2:31-113.

[2] Solomon ME (1949) The natural control of animal populations. J Anim Ecol 18:1-35.

[3] May RM, Leonard WJ (1975) Nonlinear aspects of competition between three species. SIAM J Appl Math 29(2):243-53.

[4] Murray JD (1989) Mathematical Biology. Springer

[5] Clark TJ, Luis AD (2020) Nonlinear population dynamics are ubiquitous in animals. Nat Ecol Evol 4(1):75-81.

[6] Elton CS (1924) Fluctuations in the numbers of animals: their causes and effects. British J Exp Biol 2:119-163.

[7] Elton CS, Nicholson M (1924)The 10-year cycle in numbers of the lynx in Canada. J Anim Ecol 11:215244.

[8] Schaffer WM (1984) Stretching and folding in lynx fur returns: evidence for a strange attractor in nature? Am Nat 124:798-820.

[9] Turchin P (1993) Chaos and stability in rodent population dynamics: evidence from nonlinear time-series analysis. Oikos 68:167-172.
[10] Turchin P. (1995) Chaos in microtine populations. Proc R Soc Lond B 262:357-361.

[11] Gamarra JGP, Solé RV (2000) Bifurcations and chaos in ecology: lynx returns revisited. Ecol Lett 3:114-121.

[12] Turchin P, Ellner SP (2000) Living on the edge of chaos: Population dynamics of fennoscandian voles. Ecology 81:3099-3116.

[13] Benincà E, Huisman J, Heerkloss R, Jöhnk KD, Branco P, Van Nes EH (2008) Chaos in a longterm experiment with a plankton community. Nature 451:822-826.

[14] Blasius B, Rudolf L, Weithoff G, Gaedke U, Fussmann GF (2020) Long-term cyclic persistence in an experimental predator-prey system. Nature 577(7789): 226-230.

[15] Constantino RF, Desharnais RA, Cushing JM, Dennis B (1997) Chaotic dynamics in an insect population. Science 275:389-39.

[16] Dennis B, Desharnais RA, Cushings JM, Constantino RF (1997)Estimating chaos and complex dynamics in an insect population. Anim Ecol 66:704729.

[17] Desharnais RA, Costantino RF, Cushing JM, Henson SM, Dennis B (2001) Chaos and population control of insect outbreaks. Ecol Lett 4:229-235.

[18] Dennis B, Desharnais RA, Cushing JM, Henson SM, Constantino RF (2001) Estimating Chaos and Complex Dynamics in an Insect Population. Ecol Monogr 7(12):277-303.

[19] Lenton TM, Held H, Kriegler E, Hall JW, Lucht W, Rahmstorf S, Schellnhuber H. J (2008) Tipping elements in the Earth's climate system. Proc Natl Acad Sci USA 105(6), 1786-1793.

[20] Lenton TM et al. (2019) Climate tipping points - too risky to bet against. Nature 575, 592-595.

[21] Rockström J et al (2009) A safe operating space for humanity. Nature 461:472-475.

[22] Barnosky AD et al. (2012) Approaching a state shift in Earth?s biosphere. Nature 486(7401): 52-58.

[23] Rothman DH (2017) Thresholds of catastrophe in the Earth system. Sci Adv 3(9):e1700906.

[24] Steffen W et al. (2018) Trajectories of the Earth System in the Anthropocene. Proc Natl Acad Sci USA 115(33):8252?8259.

[25] Lade, S. J., Steffen, W., de Vries, W., Carpenter, S. R., Donges, J. F., Gerten, D., Rockström, J. (2020). Human impacts on planetary boundaries amplified by Earth system interactions. Nature Sustainability, 3(2), 119?128. https://doi.org/10.1038/s41893-0190454-4

[26] Foley JA, Coe MT, Scheffer M, Wang G (2003) Regime shifts in the Sahara and Sahel: interactions between ecological and climatic systems in Northern Africa. Ecosystems 6(6): 524-532.

[27] Solé R (2007) Scaling laws in the drier. Nature 449: 151-153

[28] Berdugo M, Kéfi S, Soliveres S, Maestre FT (2017) Plant spatial patterns identify alternative ecosystem multifunctionality states in global drylands. Nat Ecol Evol 1:0003.

[29] Conde-Pueyo et al. (2020) Synthetic Biology for Terraformation Lessons from Mars, Earth, and the Microbiome. Life 10(2):14.

[30] Berdugo M, Delgado-Baquerizo M, Soliveres S, Hernández-Clemente R, Zhao Y, Gaitán JJ, Gross N, Saiz H, Maire V, Lehman A, Rillig MC, Solé R, Maestre FT (2020) Global ecosystem thresholds driven by aridity. Science 367(6479):787-790. 
bioRxiv preprint doi: https://doi.org/10.1101/2020.05.15.098863; this version posted May 16, 2020. The copyright holder for this preprint (which was not certified by peer review) is the author/funder. All rights reserved. No reuse allowed without permission.

[31] Dai L, Vorselen D, Korolev KS, Gore J (2012) Generic indicators for loss of resilience before a tipping point leading to population collapse. Science 336(6085): 1175-1177

[32] Sardanyés J, Fontich E (2010) On the metapopulation dynamics of autocatalysis: extinction transients related to ghosts. Int J Bifurc Chaos 20(4):1261-1268

[33] Sardanyés J, Solé R (2006) Ghosts in the origins of life? Int J Bifurc Chaos 16(9):2761-2765.

[34] Sardanyés J, Solé R (2007) Bifurcations and phase transitions in spatially-extended two-member hypercycles. J Theor Biol 243(4):468-482.

[35] Fontich E, Sardanyés J (2008) General scaling law in the saddle-node bifurcation: a complex phase space study. J Phys A: Math Theor 41(1):015102

[36] Vidiella B, Sardanyés J, Solé R (2018) Exploiting delayed transitions to sustain semiarid ecosystems after catastrophic shifts. J Roy Soc Interface 15(143): 20180083.

[37] Sardanyés J, Piñero J, Solé R (2019) Habitat lossinduced tipping points in metapopulations with facilitation. Pop Ecol 61(4):436-449

[38] McCann K, Yodizs P (1994) Nonlinear dynamics and population disappearances. Am Nat 144(5):873-879.

[39] Dhamala M, Lai Y-C (1999) Controlling transient chaos in deterministic flows with applications to electrical power systems and ecology. Phys Rev E 59(2): 1646-1655.

[40] Hastings A, Higgins K (1994) Persistence of transients in spatially structured ecological models. Science 263:1133.

[41] Hastings A (2004) Transients: The key long-term ecological understanding. Trends Ecol Evol 19: 3945.

[42] Morozova A, Abbott K, Cuddington K, Francis T, Gellnere G, Hastings A, Laig Y-C, Petrovskii S, Scrantonh K, LouZeemani M (2019) Long transients in ecology: theory and applications. Phys Life Rev (in press)

[43] Hastings A (2001) Transient dynamics and persistence of ecological systems. Ecol Lett 4:215-220.

[44] Hastings A, Abbott KC, Cuddington K, Francis T, Gellner G, Lai YC, Morozov A, Petrovskii S, Scranton K, Zeeman ML (2018) Transient phenomena in ecology. Science 361(6406):eaat6412.

[45] Newbold T, et al. (2016) Has land use pushed terrestrial biodiversity beyond the planetary boundary? A global assessment. Science 353(6296):288-291.

[46] Ratajczak, Z., Carpenter, S. R., Ives, A. R., Kucharik, C. J., Ramiadantsoa, T., Stegner, M. A., S Turner, M. G. (2018). Abrupt Change in Ecological Systems: Inference and Diagnosis. Trends Ecol Evol, 33(7), 513-526.

[47] Ehrlich P, Ehrlich A (1981) Extinction (Ballantine Books, New York)

[48] Simberloff D (1984) Mass extinction and the destruction of moist tropical forests. Zh Obshch Biol 45: 767-778.

[49] Wilson EO (1988) Biodiversity (National Academy, Washington DC)

[50] Tilman D, May RM, Lehman CL, Nowak MA (1994) Habitat destruction and the extinction debt. Nature, 371(6492), 65-66.

[51] Maron M, Simmonds JS, Watson JE, Sonter LJ, Bennun L, Griffiths VF, Bull JW (2020) Global no net loss of natural ecosystems. Nature Ecol Evol 4(1):46-49.

[52] Wake DB, Vredenburg VT (2008) Are we in the midst of the sixth mass extinctio? A view from the world of amphibians. Proc Natl Acad Sci USA 105:11466-11473

[53] Ceballos G, Ehrlich PR, Barnosky AD, García A, Pringle RM, Palmer TM (2015) Accelerated modern human-induced species losses: Entering the sixth mass extinction. Sci Adv 1(5):e1400253.

[54] Ceballos G, Ehrlich PR, Dirzo R (2017) Biological annihilation via the ongoing sixth mass extinction signaled by vertebrate population losses and declines. Proc Natl Acad Sci USA 114(30):E6089E6096.

[55] McCallum M L (2015) Vertebrate biodiversity losses point to a sixth mass extinction. Biodiv Conserv 24(10):2497-2519.

[56] Barnosky AD, et al. (2011) Has the Earth sixth mass extinction already arrived? Nature 471(7336):51-57.

[57] Bascompte J, Solé RV (1996) Habitat fragmentation and extinction thresholds in spatially explicit models. J Anim Ecol 65:465-473.

[58] Brooker RW et al (2008) Facilitation in plant communities: the past, the present, and the future. J Ecol 96:18-34

[59] Bruno JF, Stachowicz JJ, Bertness MD (2003) Inclusion of facilitation into ecological theory. Trends Ecol Evol 18(3): 119-125

[60] Strogatz, SH (2000) Nonlinear Dynamics and Chaos with applications to Physics, Biology, Chemistry, and Engineering. Westview Press.

[61] Trickey ST, Virgin LN (1998) Bottlenecking phenomenon near a saddle-node remnant in a Duffing oscillator. Phys Lett A 248:185-190.

[62] Carpenter S et al (2001) Trophic cascades, nutrients, and lake productivity: whole-lake experiments. Ecol Monog 71(2):163-186.

[63] Scheffer, M., Carpenter, S., Foley, J. A., Folke, C., \& Walker, B. (2001). Catastrophic shifts in ecosystems. Nature, 413(6856), 591-596.

[64] Estes JA, et al (2011) Trophic downgrading of planet Earth. Science 333(6040):301-306.

[65] Solé RV, Alonso D, McKane A (2002) Self-organised instability in complex ecosystems. Phil Trans Roy Soc Lond B, 357:667-681.

[66] Hanski I (1999) Metapopulation Ecology. Oxford Univ. Press, Oxford, UK.

[67] Strogatz SH, Westervelt RM (1989) Predicted power laws for delayed switching of charge density waves. Phys Rev B 40:10501-10508.

[68] Gomez M, Moulton DE, Vella D (2017) Critical slowing down in purely elastic ?snap-through? instabilities. Nature Phys 13:1422-146.

[69] Gil L, Balzer G, Coullet P, Dubois M, Berge, P (1991) Hopf Bifurcation in a Broken-Parity Pattern. Phys Rev Lett 66(25):3249-3252.

[70] Maselko J (1983) Bifurcation diagrams for the two substrate oscillatory systems. Chem Phys 78:381389.

[71] Strizhak P, Menzinger M (1996) Slow passage through a supercritical Hopf bifurcation: Timedelayed response in the Belousov?Zhabotinsky reaction in a batch reactor. J Chem Phys 105:10905.

[72] Hofbauer J (1994) Heteroclinic cycles in ecological differential equations. Tatra Mountains Math. Publ. 4:105-116

[73] Szolnoki A, Mobilia M, Jiang L-L, ,Szczesny B, Rucklidge AM, Perc M (2014) Cyclic dominance in evolutionary games: a review. J Roy Soc Interface 11: 20140735

[74] Tilman, D. (1994). Competition and biodiversity in spatially structured habitats. Ecology, 75(1), 2-16. 
bioRxiv preprint doi: https://doi.org/10.1101/2020.05.15.098863; this version posted May 16, 2020. The copyright holder for this preprint (which was not certified by peer review) is the author/funder. All rights reserved. No reuse allowed without permission.

[75] Kuussaari, M., Bommarco, R., Heikkinen, R. K., Helm, A., Krauss, J., Lindborg, R., ... \& Stefanescu, C. (2009). Extinction debt: a challenge for biodiversity conservation. Trends in ecology \& evolution, 24(10), 564-571.

[76] Ripple WJ, et al (2014) Status and ecological effects of the world's largest carnivores. Science 343:1241484-1-11

[77] Folke C et al (2004) Regime Shifts, Resilience, and Biodiversity in Ecosystem Management. Annual Review Ecol Evol System 35(1):557?581.

[78] Soulé M, Noss R (1998) Rewilding and Biodiversity. Wild Earth.

[79] Seddon PJ, Griffiths CJ, Soorae PS, Armstrong DP (2014) Reversing defaunation: restoring species in a changing world. Science 345(6195):406-412.

[80] Lorimer J, Sandom C, Jepson P, Doughty C, Barua M, Kirby KJ (2015) Rewilding: Science, practice, and politics. Annu Rev Environm Resources, 40:3962.

[81] Ripple WJ, Beschta RL (2012) Trophic cascades in Yellowstone: the first 15 years after wolf reintroduction. Biol. Conservation 145(1):205-213.

[82] Wolf C, Ripple WJ (2018) Rewilding the world's large carnivores. Roy Soc Open Sci 5(3):172235.

[83] Beschta RL, Ripple WJ (2016) Riparian vegetation recovery in Yellowstone: the first two decades after wolf reintroduction. Biol. Conservation 198:93-103.

[84] Strickland MS, Hawlena D, Reese A, Bradford MA, Schmitz OJ (2013) Trophic cascade alters ecosystem carbon exchange. Proc Natl Acad Sci USA 110(27): 11035-11038.

[85] Atwood TB, et al. (2015) Predators help protect carbon stocks in blue carbon ecosystems. Nature Clim Change 5(12):1038-1045.

[86] Woodroffe R, Hedges S, Durant S M (2014) To Fence or Not to Fence. Science 344(6179):46-48.

[87] Terborgh J et al (2001) Ecological meltdown in predator-free forest fragments. Science 294(5548): 1923-1926.

[88] Thurstan RH, Roberts CM (2010) Ecological Meltdown in the Firth of Clyde, Scotland: Two Centuries of Change in a Coastal Marine Ecosystem. PLoS ONE 5(7):e11767.

[89] Solé RV, Montoya JM (2006) Ecological network meltdown from habitat loss and fragmentation. In Ecological Networks: Linking Structure to Dynamics in Food Webs (Eds. Pascual M, Dunne JA). Santa Fe studies in the science of complexity pages 305-323.

[90] Lindenmayer DB, Nix HA (1993) Ecological principles for the design of wildlife corridors. Conserv Biol $7(3), 627-631$.

[91] Newmark WD (1993) The role and design of wildlife corridors with examples from Tanzania. Ambio, 500504.

[92] Kiffner C, Nagar S, Kollmar C, Kioko J (2016) Wildlife species richness and densities in wildlife corridors of Northern Tanzania. J. Nature Conserv. 31:29-37.

[93] Holling CS (1959) The components of predation as revealed by a study of small mammal predation of the European pine sawfly. Canadian Entom. 91:293320 .

[94] Holling CS (1959) Some characteristics of simple types of predation and parasitism. Canadian Entom 91:385-398.

[95] Holling CS (1965) The functional response of predators to prey density and its role in mimicry and population regulation. Memoirs Entom Soc Canada 45:5-
60.

[96] Smout S, Asseburg C, Matthiopoulos J, Fernandez C, Redpath S, Thirgood S, Harwood J (2010) The functional response of a generalist predator. PLoS ONE 5:e10761

[97] Rosenzweig ML, MacArthur RH (1963) Graphical representation and stability conditions of predatorprey interactions. Am Nat 97:209-23.

[98] Veraart AJ et al (2012) Recovery rates reflect distance to a tipping point in a living system. Nature 481(7381):357-359.

[99] Bernardino, P. N., De Keersmaecker, W., Fensholt, R., Verbesselt, J., Somers, B., \& Horion, S. (2020). Global-scale characterisation of turning points in arid and semi-arid ecosystem functioning. Global Ecology and Biogeography.

[100] Mollmann C, Diekmann R (2012) Marine ecosystem regime shifts induced by climate and overfishing: a review for the Northern Hemisphere. In Advances in Ecological Research (Vol. 47, pp. 303-347). Academic Press.

[101] Saleem M (2015) Loss of microbiome ecological niches and diversity by global change and trophic downgrading. In Microbiome Community Ecology (pp. 89-113). Springer, Cham.

[102] Dirzo, R., Young, H. S., Galetti, M., Ceballos, G., Isaac, N. J. B., \& Collen, B. (2014). Defaunation in the Anthropocene. In Science (Vol. 345). https://doi.org/10.1126/science.1251817

[103] Young, H. S., McCauley, D. J., Galetti, M., \& Dirzo, R. (2016). Patterns, Causes, and Consequences of Anthropocene Defaunation. Annual Review of Ecology, Evolution, and Systematics, 47(1), 333-358. https://doi.org/10.1146/annurevecolsys-112414-054142

[104] Ellis EC (2015) Ecology in an anthropogenic biosphere. Ecol Monographs 85(3):287-331.

[105] Svenning JC, et al (2016) Science for a wilder Anthropocene: Synthesis and future directions for trophic rewilding research. Proc Natl Acad Sci USA 113(4):898-906

[106] Johnson CN, Balmford A, Brook BW, Buettel JC, Galetti M, Guangchun L, Wilmshurst JM (2017) Biodiversity losses and conservation responses in the Anthropocene. Science 356(365):270-275

[107] Bowler DE et al (2020) Mapping human pressures on biodiversity across the planet uncovers anthropogenic threat complexes. People and Nature 00:115.

[108] Sardanyés J, Duarte J, Januário C, Martins N (2012) Controlling delayed transitions with applications to prevent single species extinctions. Adv Diff Eq Control Proc 10(1):29-41.

[109] Bello, C., Galetti, M., Pizo, M. A., Magnago, L. F. S., Rocha, M. F., Lima, R. A. F. F., ... Jordano, P. (2015). Defaunation affects carbon storage in tropical forests. Science Advances, 1(11), 1-11. https://doi.org/10.1126/sciadv.1501105

[110] Gardner, C. J., Bicknell, J. E., BaldwinCantello, W., Struebig, M. J., \& Davies, Z. G. (2019). Quantifying the impacts of defaunation on natural forest regeneration in a global meta-analysis. Nature Communications, 10(1), 4590. https://doi.org/10.1038/s41467-019-12539-1

[111] Buzhdygan OY, et al. (2020) Biodiversity increases multitrophic energy use efficiency, flow and storage in grasslands. Nature Ecol Evol 4:393-405

[112] Kuznetsov, Yuri A. Elements of applied bifurcation theory. Second edition. Applied Mathematical Sci- 
bioRxiv preprint doi: https://doi.org/10.1101/2020.05.15.098863; this version posted May 16, 2020. The copyright holder for this preprint (which was not certified by peer review) is the author/funder. All rights reserved. No reuse allowed without permission.

ences, 112. Springer-Verlag, New York, 1998.

[113] Shilnikov LP, Shilnikov AL, Turaev D, Chua LO (20019) Methods of qualitative theory in nonlinear dynamics. Part II. World Scientific Series on Nonlinear Science. Series A: Monographs and Treatises, 5. World Scientific Publishing Co., Inc., River Edge, NJ.

[114] All numerical results have been obtained with a Runge-Kutta-Fehlberg method of $7^{\text {th }}-8^{t h}$ order with automatic step size control and local relative tolerance $10^{-15}$.

[115] Cycles are considered as the returning time to $V(t)=$ $\kappa / \epsilon_{1}$ once this threshold is crossed twice by a given orbit (see Section IV B in the Appendix).

\section{APPENDIX}

\section{A. Heteroclinic bifurcation}

For the convenience of the reader, in this appendix we describe the key points of the heteroclinic bifurcation which takes place in Eqs. (6)-(7). It is similar to the Andronov-Leontovich homoclinic bifurcation, see for instance $[112,113]$. Let us write our $2 D$ system on a generic form as $\dot{z}=X(z, p), z=(x, y)$ or

$$
\begin{aligned}
& \dot{x}=f(x, y, p) \\
& \dot{y}=g(x, y, p),
\end{aligned}
$$

where $p$ is a parameter. First, we present the setting of the heteroclinic bifurcation we deal with. The line $\{y=0\}$ is invariant and contains two equilibrium points $P_{1}=\left(x_{1}, 0\right), P_{2}=\left(x_{2}, 0\right)$ of saddle type with $0<x_{1}<x_{2}$. The position of $P_{1}, P_{2}$ may depend on the parameter $p$. Let $\lambda_{i}, \mu_{i}$ (here with $i=1,2)$, with $\lambda_{i}<0<\mu_{i}$ be the eigenvalues of $\mathrm{D} X\left(P_{i}, p\right)$. In general these eigenvalues depend on $p$. The unstable invariant manifold of $P_{1}$, labeled $W^{u}\left(P_{1}\right)$, and the stable invariant manifold of $P_{2}$, labeled $W^{s}\left(P_{2}\right)$, are contained in $\{y=0\}$. Let $\sigma_{1}$ be the corresponding heteroclinic connection between $P_{1}$ and $P_{2}$. Given $x_{N} \in\left(x_{1}, x_{2}\right)$, the branches of $W^{s}\left(P_{1}\right)$ and $W^{u}\left(P_{2}\right)$ in the region $\{x \geq 0, y \geq 0\}$ cross the section $\left\{x=x_{N}\right\}$ at points $Q^{s}(p)$ and $Q^{u}(p)$ respectively. There exists a value of the parameter $p=p_{0}$ such that $Q^{s}\left(p_{0}\right)=Q^{u}\left(p_{0}\right)$. Let

$$
\Delta=\frac{d}{d p}\left(Q^{s}(p)-Q^{u}(p)\right)_{\left.\right|_{p=p_{0}}} .
$$

Let $\sigma_{2}$ be the heteroclinic connection along the mentioned branches when $p=p_{0}$. Now we assume two more specific conditions

a) $\frac{\lambda_{1} \mu_{2}}{\mu_{1} \lambda_{2}}>1$

b) $\Delta>0$.

Then, there exists a neighbourhood $U$ of the heteroclinic cycle $\sigma_{h}=\sigma_{1} \cup \sigma_{2} \cup\left\{P_{1}, P_{2}\right\}$ and an interval $\left(p_{-}, p_{+}\right)$with $p_{0} \in\left(p_{-}, p_{+}\right)$such that for $p \in\left(p_{0}, p_{+}\right)$ there exists a unique periodic orbit $\gamma_{p}$ of system (32) in $U$ which is asymptotcially stable. Moreover, $\gamma_{p}$ tends to $\sigma_{h}$ when $p \rightarrow p_{0}$. For $p \in\left(p_{-}, p_{0}\right)$ system $(32)$ has no periodic orbits in $U$.

To prove the claim we introduce a return map close to $\sigma_{h}$ for values of $p$ close to $p_{0}$. Since our vector field is analytic there exists $C^{1}$ local changes of coordinates $h_{1}$ and $h_{2}$ near $P_{1}$ and $P_{2}$, respectively, which (locally) linearize the system. Let $V_{1}, V_{2}$ be the domains of $h_{1}, h_{2}$. We take sections $\Sigma_{1}^{-}, \Sigma_{1}^{+}$transversal to the flow in $V_{1}$ which are preimages by $h_{1}$ of the sections $\Gamma_{1}^{-}=\left\{(\xi, \eta) \mid \eta=\eta_{1}\right\} \cap h_{1}\left(V_{1}\right)$ and $\Gamma_{1}^{+}=\{(\xi, \eta) \mid \xi=$ $\left.\xi_{1}\right\} \cap h_{1}\left(V_{1}\right)$ respectively, as shown in the image below.
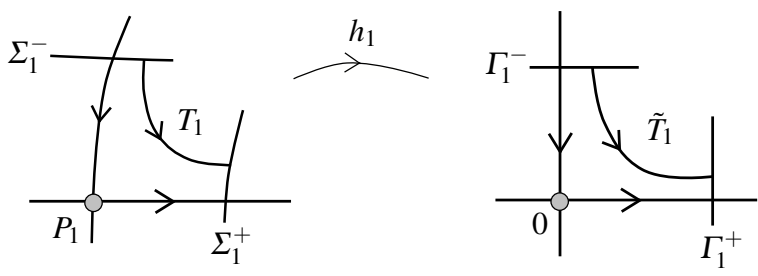

We define $\tilde{T}_{1}$ as the map which sends the coordinate $\xi$ of the point $\left(\xi, \eta_{1}\right) \in \Gamma_{1}^{-}$to the coordinate $\eta$ of the point $\left(\xi_{1}, \eta\right) \in \Gamma_{1}^{+}$obtained as the intersection of the orbit of $\left(\xi, \eta_{1}\right)$ of the linear flow with $\Gamma_{1}^{+}$.

The linearised system reads

$$
\begin{aligned}
& \dot{\xi}=\mu_{1} \xi \\
& \dot{\eta}=\lambda_{1} \eta .
\end{aligned}
$$

An easy computation from the general solution $\xi(t)=$ $\xi(0) e^{\mu_{1} t}, \eta(t)=\eta(0) e^{\lambda_{1} t}$, gives the time to arrive from $\left(\xi, \eta_{1}\right)$ to $\Gamma_{1}^{+}$:

$$
t=\frac{1}{\mu_{1}} \log \left(\frac{\xi_{1}}{\xi}\right)
$$

and then

$$
\eta=C\left(\xi_{1}, \eta_{1}\right) \xi^{-\lambda_{1} / \mu_{1}}, \quad C\left(\xi_{1}, \eta_{1}\right)=\eta_{1} \xi_{1}^{\lambda_{1} / \mu_{1}} .
$$

Going back by $h^{-1}$ to $(x, y)$ variables, using $\xi, \eta$ as coordinates in $\Sigma_{1}^{-}$and $\Sigma_{1}^{+}$respectively, we have

$$
\eta=T_{1}(\xi)=C_{1} \xi^{-\lambda_{1} / \mu_{1}} .
$$

In a completely analogous way we define the map $T_{2}$ from $\Sigma_{2}^{-}$to $\Sigma_{2}^{+}$, where $\Sigma_{2}^{-}$and $\Sigma_{2}^{+}$are the preimages by $h_{2}$ of $\Gamma_{2}^{-}=\left\{(\xi, \eta) \mid \xi=\xi_{2}\right\} \cap h_{2}\left(V_{2}\right)$ and $\Gamma_{2}^{+}=$ $\left\{(\xi, \eta) \mid \eta=\eta_{2}\right\} \cap h_{2}\left(V_{2}\right)$ respectively. See the diagram below.

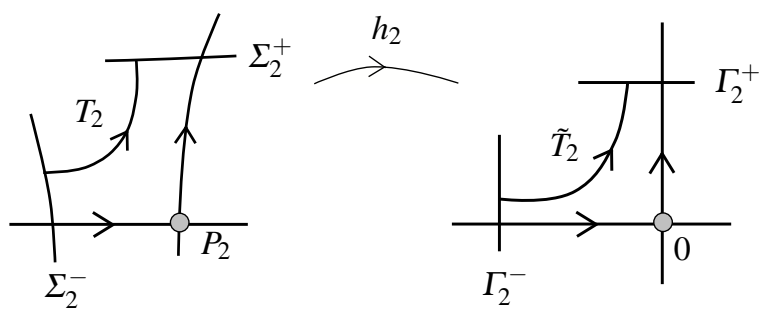

In local coordinates $u$ in $\Sigma_{2}^{-}$and $v$ in $\Sigma_{2}^{+}$

$$
v=T_{2}(u)=C_{2} u^{-\mu_{2} / \lambda_{2}},
$$


where $C_{2}$ only depends on the chosen sections (and the parameter).

Also, we define $S_{1}: \Sigma_{1}^{+} \longrightarrow \Sigma_{2}^{-}$and $S_{2}: \Sigma_{2}^{+} \longrightarrow$ $\Sigma_{1}^{-}$as regular Poincaré maps among the corresponding sections. They are analytic diffeomorphisms. Since the line $\{y=0\}$ is invariant for all values of the parameter, $S_{1}(0, p)=0$ and therefore

$$
\begin{aligned}
S_{1}(\eta, p) & =\eta \hat{S}_{1}(\eta, p) \\
& =\eta\left[a_{10}+a_{20} \eta+a_{11}\left(p-p_{0}\right)+\mathcal{O}_{2}\left(\eta, p-p_{0}\right)\right]
\end{aligned}
$$

As for $S_{2}$, we have $S_{2}\left(0, p_{0}\right)=0$. The condition $\Delta>0$ implies

$$
b_{01}=\frac{\partial S_{2}}{\partial p}\left(0, p_{0}\right)>0,
$$

and then

$$
S_{2}(v, p)=b_{10} v+b_{01}\left(p-p_{0}\right)+\mathcal{O}_{2}\left(v, p-p_{0}\right) .
$$

Since $S_{1}, S_{2}$ are diffeomorphisms we have $a_{10}, b_{10} \neq 0$. Moreover, since we are in the plane, the maps preserve orientation and therefore $a_{10}, b_{10}>0$. Then the return map $R: \Sigma_{1}^{-} \longrightarrow \Sigma_{1}^{-}$defined for $\xi>0$ and $p-p_{0}$ small as $R=S_{2} \circ T_{2} \circ S_{1} \circ T_{1}$ has the form

$$
\begin{aligned}
R(\xi)= & b_{10} C_{2} C_{1}^{-\mu_{2} / \lambda_{2}} a_{10}^{-\mu_{2} / \lambda_{2}}\left(\xi^{\lambda_{1} \mu_{2} / \mu_{1} \lambda_{2}}+\right. \\
& \left.\mathcal{O}_{1}\left(\xi^{-\lambda_{1} / \mu_{1}}, p-p_{0}\right)\right)+b_{01}\left(p-p_{0}\right)+ \\
& \mathcal{O}_{2}\left(\xi^{\lambda_{1} \mu_{2} / \mu_{1} \lambda_{2}}, p-p_{0}\right) .
\end{aligned}
$$

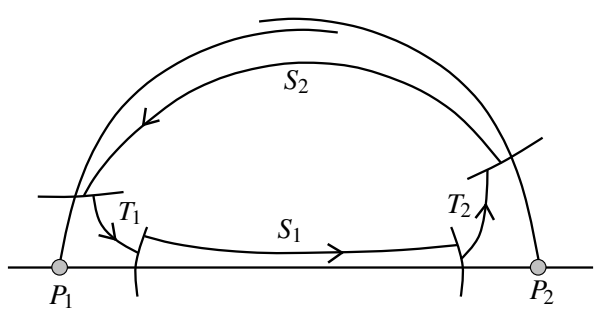

$R$ is a perturbation of the $1 D$ map

$$
R_{0}(\xi)=A \xi^{\lambda_{1} \mu_{2} / \mu_{1} \lambda_{2}}+B\left(p-p_{0}\right)
$$

with $A, B>0$ and $\lambda_{1} \mu_{2} /\left(\mu_{1} \lambda_{2}\right)>1$. We display the graphs of $R_{0}$ for $p<p_{0}, p=p_{0}$, and $p>p_{0}$.



The claim follows directly from the interpretation of the image above. Indeed, notice that graph $R_{0}$ cuts the diagonal close to $\xi=0$ in and only if $p>$ $p_{0}$. The intersection point belongs to a periodic orbit. Moreover, since graph $R_{0}$ crosses $\{y=x\}$ from the upper to the lower part of the diagonal, the periodic orbit is asymptotically stable. Also, the fixed point tends to $\xi=0$ when $p$ tends to $p_{0}$.

\section{B. Computation of the heteroclinic bifurcation value}

Here we explain how the heteroclinic bifurcation value has been obtained by means of a numerical study of the global manifolds. The basic idea is to track when the STABLE MANIFOLD of the upper branch of equilibrium $P_{1-}^{*}$ coincides with the UNSTABLE MANIFOLD of the upper branch of $P_{1+}^{*}$. These two points have a HETEROCLINIC CONNECTION at bifurcation value. Before the bifurcation, a separation between the manifolds exists. After the bifurcation, a gap between the two manifolds will involve that all orbits go to the origin. Let us use the vegetation-herbivore model to illustrate the followed procedure (setting $\mu=1$ for simplicity and using $\kappa=\delta / \mu=\delta)$ :

$$
\begin{aligned}
\frac{d V}{d t} & =\alpha V^{2}(1-D-V)-\epsilon V-\epsilon_{1} V S_{1} \\
\frac{d S_{1}}{d t} & =\epsilon_{1} V S_{1}-\kappa S_{1}
\end{aligned}
$$

The basic procedure consists of:

1. Identify the equilibrium points that can get connected by a heteroclinic manifold. Here, there are three equilibria: the interior equilibrium $\left(P_{2}^{*}\right.$, repeller focus) and two saddle points $\left(P_{1 \pm}^{*}\right)$.
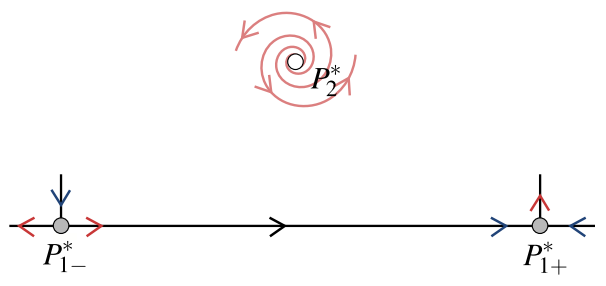

2. Calculate the eigenvectors $\vec{v}_{P^{*}}$ for each equilibrium point $P^{*}$ that become heteroclinically connected i.e., the unstable manifold of one equilibrium becomes the same as the stable manifold of the other, here for $P_{1-}^{*}$ and $P_{1+}^{*}$. These eigenvectors read:

$$
\begin{aligned}
& \vec{v}_{P_{1-}^{*}}=\left(1,-1+\frac{(1-D) \alpha \kappa+(2 \epsilon+\kappa) \sqrt{(1-D)^{2} \alpha^{2}-4 \alpha \epsilon}}{2 \epsilon \epsilon_{1}}\right) \\
& \vec{v}_{P_{1+}^{*}}=\left(1,-1+\frac{(1-D) \alpha \kappa-(2 \epsilon+\kappa) \sqrt{(1-D)^{2} \alpha^{2}-4 \alpha \epsilon}}{2 \epsilon \epsilon_{1}}\right)
\end{aligned}
$$

3. Compute the unstable manifold, $W^{u}$. To do so, integrate the ODEs numerically using as initial condition $P_{1+}^{*}+\xi \vec{v}_{P_{1+}^{*}}$ (using small $\xi$, here $10^{-5}$ ).

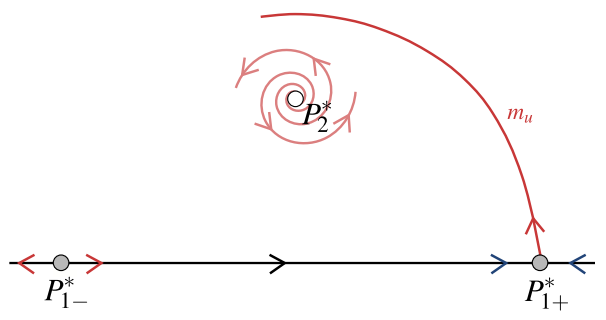

4. Compute the stable manifold, $W^{s}$. To do so, we compute the solution of the ODEs numerically using as initial condition $P_{1-}^{*}+\xi \vec{v}_{P_{1-}^{*}}$. In this case, the solutions are computed in backward time. Since 
this manifold is stable, it attracts the orbits and the direction of the field needs to be changed to globalize the manifold.

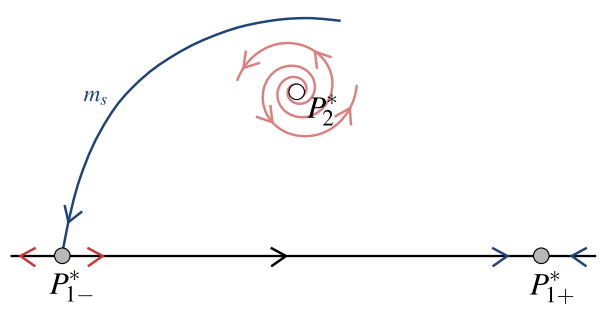

5. Chose a place (in the phase space) where the distance between the manifolds can be computed. Once the two manifolds collide they will collide simultaneously in all their trajectory (since they are heteroclinically connected). In the case shown in the main text, the value chosen was $V=\kappa / \varepsilon_{1}$, the first time that this value is achieved. Let $m_{u}$ and $m_{s}$ be the intersection of $W^{u}$ and $W^{s}$ (for the first time) with the line $\kappa / \varepsilon_{1}$ for first time.

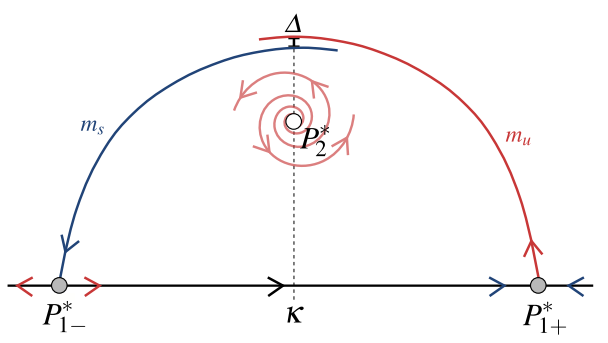

6. Compute the diference between the stable and unstable manifolds $\left(\Delta=m_{u}-m_{s}\right)$, while changing the parameter for which the critical value is desired. At some point, the distance will become negative, then the heteroclinic bifurcation had occured.
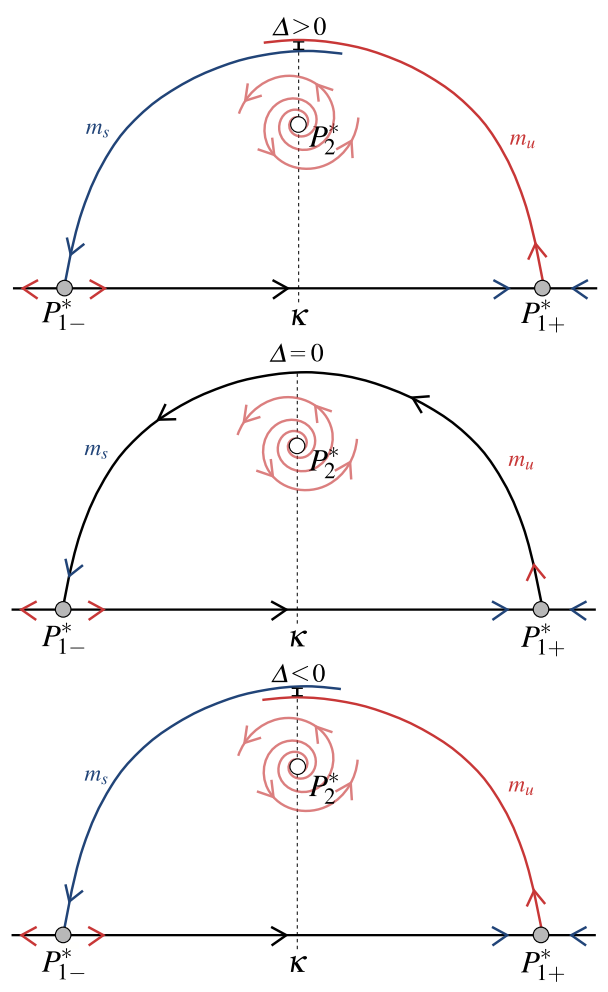

For the model studied in this article this procedure can be done in an easier manner. Knowing that the unstable manifold can only end-up going to the extinction fixed point (before the bifurcation), or to the interior fixed point (coexistence of vegetation and herbivores, after the bifurcation) the procedure can be simplified. The bifurcation value will be the parameter value for which there is a change between going to the extinction point or not. 